\title{
Excess Thermodynamic Properties of Mixtures Involving Xenon and Light Alkanes: A Study of Their Temperature Dependence by Computer Simulation
}

\author{
Luís F. G. Martins, ${ }^{*+}$ A. J. Palace Carvalho, ${ }^{\dagger}$ J. P. Prates Ramalho, $^{\dagger}$ and Eduardo J. M. Filipe ${ }^{\ddagger}$ \\ ${ }^{+}$Centro de Química de Évora, University of Évora, Rua Romão Ramalho, 59, 7000-671 Évora, Portugal \\ ${ }^{\ddagger}$ Centro de Química Estrutural, Instituto Superior Técnico, Avenida Rovisco Pais, 1049-001, Lisbon, Portugal
}

ABSTRACT: As a natural extension of a previous work, excess molar enthalpies and excess molar volumes as a function of composition in a wide range of temperatures have been obtained for binary mixtures of xenon with ethane, propane, and $n$-butane by Monte Carlo computer simulation. Xenon was modeled by a simple spherical Lennard-Jones potential, and the TraPPE-UA force field was used to describe the $n$-alkanes. One of the main goals of this study is to investigate the temperature dependence of the excess properties for mixtures of xenon and $n$-alkanes and, if possible, to supplement the lack of experimental data. For all three

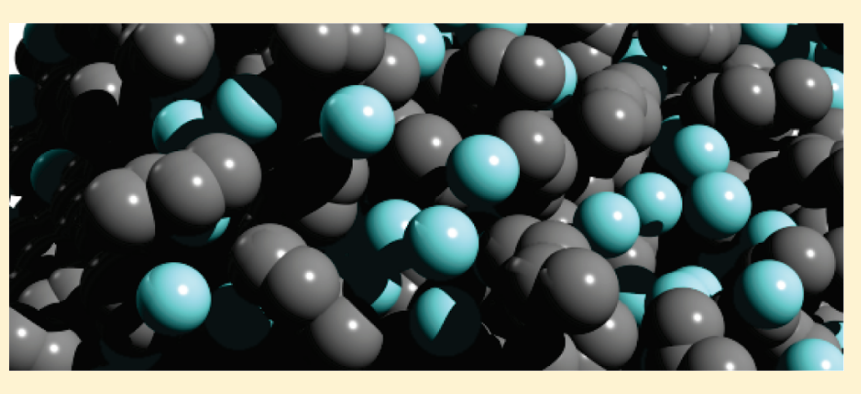
systems, the simulation results predicted excess volumes in good agreement with the experimental data. As for the excess enthalpies, in the case of (xenon + ethane), the simulation results confirm the negative experimental result and the weak temperature dependence. In the case of (xenon + propane) and (xenon $+n$-butane), however, the simulation predicts negative excess enthalpies, but those estimated from experimental data are positive. Both excess volumes and enthalpies display a complex dependence on temperature that in some aspects resembles that found for mixtures of $n$-alkanes.The structure of the liquid mixtures was also investigated by calculating radial distribution functions $\left[g_{\alpha \beta}(r)\right]$ between each pair of interaction groups for all the binary systems at all temperatures. It is found that the mean distance between xenon and $\mathrm{CH}_{2}$ groups is systematically higher than the distance between xenon and $\mathrm{CH}_{3}$. In addition, the number of groups around xenon in the first coordination sphere was calculated and seems to be proportionally more populated by methyl groups than by methylene groups. The results seem to reflect a preferential and stronger interaction between xenon and $\mathrm{CH}_{3}$, in agreement with previous findings.

\section{INTRODUCTION}

The study of binary mixtures of light $n$-alkanes with noble gases remains nowadays a useful tool to understand molecular interactions at a fundamental level and the role they play in macroscopic properties of liquid mixtures. This knowledge can, in turn, be extended to the theoretical description of systems of much more complexity. Given their important applications, in particular in the fields of energy and power, the $n$-alkanes are probably the most studied chemical family in terms of thermodynamic properties and regularity of their physical properties. However, the lightest $n$-alkanes are known to deviate from that regularity, ${ }^{1}$ and understanding this transition is an important fundamental subject.

Despite its rarity, structural simplicity and price, xenon is presently used in many different applications, such as lighting, laser technology, or as a filler gas in plasma display panels. However, some of the most interesting and promising applications of xenon are in the field of medicine, such as an imaging agent in magnetic resonance, as a filler gas in nonlaser lamps used in photodynamic therapy, ${ }^{3}$ and mainly as an inhalation anesthetic agent. ${ }^{4}$ Xenon has been considered a perfect anesthetic because it produces anesthesia at normal pressure with minimal side effects: ${ }^{5}$ its use is characterized by a remarkable cardiovascular stability, ${ }^{6}$ rapid onset and offset of its action (due to its low bloodgas coefficient), ${ }^{7}$ and neuroprotection. ${ }^{7,8}$ Furthermore, there is no indication of toxic, mutagenic, or carcinogenic effects of this practice. ${ }^{7}$ The high price of xenon is the main disadvantage of its generalized use as an anesthetic, which needs to be overcome by developing efficient recycling devices. ${ }^{9}$

Although the molecular mechanism of anesthesia is still a matter of controversy, ${ }^{4}$ it is generally accepted that xenon interacts with either the lipid bilayers of nerve cell membranes, ${ }^{10-12}$ changing their physiologic properties, or with specific hydrophobic pockets in proteins that consist of neurotransmitter receptor sites and ion channels. ${ }^{13,14,4,15}$ In either case, the study of mixtures involving xenon and alkanes (in particular, the change of properties upon mixing) can therefore contribute to shedding some light on the molecular basis of this effect. Furthermore, given the simplicity of the components and interactions, these mixtures can also be used as models for understanding the behavior of more complex systems.

Received: March 21, 2011

Revised: June 1, 2011

Published: July 03, 2011 
It is known that $n$-alkanes mix with positive excess enthalpies at low temperature, which decrease with increasing temperature, passing through zero, and become negative at higher temperatures. ${ }^{16-28}$ The temperature at which excess molar enthalpy, $H_{\mathrm{m}}^{\mathrm{E}}(x=0.5)$ becomes negative and changes from system to system. Interestingly, if the same data are plotted against the reduced temperature of the mixtures, it is found that the temperature at which $H_{\mathrm{m}}^{\mathrm{E}}(x=0.5)$ becomes negative is practically the same for all systems, falling within $0.57-0.59 .^{21}$ Blas and dos Ramos ${ }^{22}$ were able to theoretically reproduce this universal behavior using the soft-SAFT equation and suggested that the same trends should exist for other excess properties (e.g., excess molar volume, $\left.V_{\mathrm{m}}^{\mathrm{E}}\right)$ and compositions. Checking the existence of that universal behavior for mixtures of xenon with the lightest $n$-alkanes is the main goal of this work and an interesting fundamental challenge. In fact, in previous work, we have shown that mixtures of xenon and $n$-alkanes exhibit a behavior that closely resembles that seen for mixtures of $n$-alkanes.

Our research group has reported experimental thermodynamic studies of mixtures of xenon with some of the lightest alkanes such as ethane, propane, and $n$-butane at low temperatures. Excess Gibbs energies and excess molar volumes were measured for all these systems ${ }^{23,24}$ and found to be negative, decreasing with the increasing temperature and $n$-alkane chain length. Since mixtures of $n$-alkanes also display negative excess Gibbs energies and volumes, these results were seen as a confirmation of the resemblance between the two types of mixtures.

However, excess molar enthalpies have been calorimetrically measured only for (xenon + ethane) at one temperature, $\left[H^{\mathrm{E}}(x=0.5)=-51.7 \mathrm{~J} \cdot \mathrm{mol}^{-1}\right]$, but for $($ xenon + propane $)$ and (xenon $+n$-butane) they were estimated from the temperature dependence of the excess Gibbs energy and found to be positive $\left(H^{\mathrm{E}}(x=0.5)=+138 \mathrm{~J} \cdot \mathrm{mol}^{-1}\right.$ and $H^{\mathrm{E}}(x=0.5)=+17 \mathrm{~J} \cdot \mathrm{mol}^{-1}$, respectively). Clearly, this amount of experimental data is not sufficient to establish the similarity between (xenon $+n$-alkanes) and ( $n$-alkanes $+n$-alkanes) in terms of excess enthalpies. In particular, the positive excess enthalpies estimated for (xenon + propane) and (xenon $+n$-butane) are difficult to interpret unless it is proven that they become negative at a higher temperature.

Using the semiempirical Deiters equation of state, we were recently able to reproduce the temperature dependence of the excess enthalpies for mixtures of $n$-alkanes, previously described. ${ }^{25}$ Moreover, the equation predicts similar temperature dependence for mixtures of (xenon $+n$-alkanes). In particular, the reduced temperature at which $H^{\mathrm{E}}(x=0.5)$ becomes zero is approximately the same for both types of systems. This was a very encouraging result to carry out the present study.

From a theoretical point of view, the experimental data for (xenon $+n$-alkane) mixtures, as interpreted by the statistical associating fluid theory, ${ }^{26}$ showed that xenon can be represented by a sphere with almost the same diameter and intermolecular potential as those suited to describe the $n$-alkanes. The diameter of the xenon atom (measured, for instance, in terms of its van der Waals radius) agrees very well with that of the cross-sectional diameter of the $n$-alkanes. Interestingly, it was shown that this resemblance extends to anesthesia. ${ }^{26}$ Minimum alveolar concentration (MAC) of $n$-alkanes, as inhalation anesthetics, varies regularly with chain length, with methane being a notable exception. Xenon follows the general behavior of $n$-alkanes, replacing methane in the beginning of the $n$-alkane series.

Further examples of the similarity between the behavior of (xenon $+n$-alkane $)$ and the $(n$-alkane $+n$-alkane $)$ interactions
Table 1. Parameters of United Atom Intermolecular Interactions

\begin{tabular}{lcl} 
& \multicolumn{2}{c}{ Lennard-Jones parameters } \\
\cline { 2 - 3 } & $\varepsilon / \mathrm{K}$ & $\sigma / \AA$ \\
$\mathrm{CH}_{2}$ & 46 & 3.95 \\
$\mathrm{CH}_{3}$ & 98 & 3.75 \\
$\mathrm{Xe}$ & 227.8557 & 3.9478 \\
\hline
\end{tabular}

Table 2. Parameters of Alkane Intramolecular Parameters

\begin{tabular}{lll} 
& \multicolumn{2}{c}{ intramolecular parameters } \\
bond terms & $r_{0}$ & $1.54 \AA$ \\
angle terms & $c_{1}$ & $31250 \mathrm{~K} / \mathrm{rad}^{2}$ \\
& $\theta_{0}$ & $114^{\circ}$ \\
torsion terms & $c_{1}$ & $355.03 \mathrm{~K}$ \\
& $c_{2}$ & $-68.19 \mathrm{~K}$ \\
& $c_{3}$ & $791.32 \mathrm{~K}$ \\
\hline
\end{tabular}

were provided by two recent works from our research group. In the first one, the solubility of xenon in $n$-pentane and $n$-hexane was measured as a function of temperature, ${ }^{27}$ from which solvation enthalpies, solvation entropies, and interaction enthalpies $\left(H_{\text {int }}\right)$ were estimated. We have found that a plot of $H_{\text {int }}$ vs $T_{\mathrm{r}}$ shows a maximum at $T_{r}=0.57$ for both (xenon $+n$-pentane) and (xenon $+n$-hexane). Interestingly, this reduced temperature is very close to that at which $H_{\mathrm{m}}^{\mathrm{E}}(x=0.5)$ vanishes for binary mixtures of $n$-alkanes $(0.57-0.59)$. In the second one, ${ }^{28}$ we have been able to decompose the interaction enthalpy into its (xenon + $\mathrm{CH}_{2}$ ) and (xenon $+\mathrm{CH}_{3}$ ) contributions, confirming that the $\mathrm{Xe}-\mathrm{CH}_{3}$ interaction is stronger than $\mathrm{Xe}-\mathrm{CH}_{2}$.

We have recently reported Monte Carlo computer simulations of excess molar enthalpies and excess molar volumes for the binary mixtures of xenon with ethane, propane, and $n$-butane at low temperatures. ${ }^{29}$ The simulation results presented an excellent agreement with experimental ones for (xenon + ethane), whereas for the remaining systems, the existing experimental results of excess molar volumes were reproduced within $50 \%$, which can be considered very good. However, the simulations were performed at a single temperature for each system. In this work, we have extended the previous study obtaining Monte Carlo simulations results for the excess properties of the same systems in a wide range of temperatures (160-255 K). Structural information has also been obtained by calculating radial distribution functions for each pair of interactive groups present in the molecules. The results provide a much clearer picture of the structure and underlying interactions of liquid (xenon + n-alkane) mixtures.

\section{SIMULATION DETAILS}

The computer simulations both for pure fluids and liquid mixtures were performed using Monte Carlo method, using the MCCCS Towhee Monte Carlo molecular simulation package, version 5.2.12 (http://towhee.sourceforge.net/). TraPPE-UA ${ }^{30}$ was used as the force field for the $n$-alkanes. This force field uses fixed bond lengths, harmonic style angle bending terms, and quadratic torsion terms for the intramolecular interactions, whereas the intermolecular terms are modeled by Lennard-Jones 
Table 3. Simulation Results of Excess Molar Enthalpy and Excess Molar Volume for Mixtures of Xenon with Ethane As a Function of Composition at Several Temperatures, along with System Pressure and Number of Molecules of Each Component for All the Simulations

\begin{tabular}{|c|c|c|c|c|c|}
\hline$x(\mathrm{Xe})$ & $p / \mathrm{kPa}$ & $N_{\mathrm{Xe}}$ & $N_{\text {ethane }}$ & $H_{\mathrm{m}}^{\mathrm{E}} / \mathrm{J} \cdot \mathrm{mol}^{-1}$ & $V_{\mathrm{m}}^{\mathrm{E}} / \mathrm{cm}^{3} \cdot \mathrm{mol}^{-1}$ \\
\hline \multicolumn{6}{|c|}{$T=161.40 \mathrm{~K}$} \\
\hline 0 & 24 & 0 & 500 & 0 & 0 \\
\hline 0.2 & 35 & 100 & 400 & $-21.9 \pm 9.4$ & $-0.041 \pm 0.031$ \\
\hline 0.4 & 46 & 300 & 450 & $-33.6 \pm 8.2$ & $-0.062 \pm 0.026$ \\
\hline 0.5 & 52 & 350 & 350 & $-35.3 \pm 8.3$ & $-0.066 \pm 0.027$ \\
\hline 0.6 & 58 & 510 & 340 & $-35.2 \pm 7.9$ & $-0.065 \pm 0.025$ \\
\hline 0.8 & 70 & 800 & 200 & $-24.2 \pm 7.7$ & $-0.046 \pm 0.023$ \\
\hline 1 & 83 & 900 & 0 & 0 & 0 \\
\hline \multicolumn{6}{|c|}{$T=170 \mathrm{~K}$} \\
\hline 0 & 45 & 0 & 500 & 0 & 0 \\
\hline 0.2 & 66 & 100 & 400 & $-20.9 \pm 7.5$ & $-0.041 \pm 0.025$ \\
\hline 0.4 & 80 & 300 & 450 & $-31.4 \pm 8.0$ & $-0.063 \pm 0.027$ \\
\hline 0.5 & 90 & 350 & 350 & $-34.9 \pm 8.2$ & $-0.069 \pm 0.027$ \\
\hline 0.6 & 100 & 510 & 340 & $-35.2 \pm 8.4$ & $-0.069 \pm 0.028$ \\
\hline 0.8 & 120 & 800 & 200 & $-23.1 \pm 6.8$ & $-0.044 \pm 0.022$ \\
\hline 1 & 140 & 900 & 0 & 0 & 0 \\
\hline \multicolumn{6}{|c|}{$T=182.34 \mathrm{~K}$} \\
\hline 0 & 95 & 0 & 500 & 0 & 0 \\
\hline 0.2 & 120 & 100 & 400 & $-21.2 \pm 7.1$ & $-0.044 \pm 0.026$ \\
\hline 0.4 & 150 & 300 & 450 & $-31.7 \pm 6.4$ & $-0.064 \pm 0.024$ \\
\hline 0.5 & 165 & 350 & 350 & $-34.4 \pm 7.1$ & $-0.073 \pm 0.026$ \\
\hline 0.6 & 185 & 510 & 340 & $-31.2 \pm 7.0$ & $-0.061 \pm 0.025$ \\
\hline 0.8 & 215 & 800 & 200 & $-24.0 \pm 6.1$ & $-0.051 \pm 0.022$ \\
\hline 1 & 250 & 900 & 0 & 0 & 0 \\
\hline \multicolumn{6}{|c|}{$T=195.49 \mathrm{~K}$} \\
\hline 0 & 180 & 0 & 500 & 0 & 0 \\
\hline 0.2 & 230 & 100 & 400 & $-20.8 \pm 10.1$ & $-0.050 \pm 0.040$ \\
\hline 0.4 & 285 & 300 & 450 & $-30.2 \pm 7.8$ & $-0.072 \pm 0.032$ \\
\hline 0.5 & 310 & 350 & 350 & $-34.7 \pm 8.2$ & $-0.093 \pm 0.034$ \\
\hline 0.6 & 335 & 510 & 340 & $-32.9 \pm 7.9$ & $-0.083 \pm 0.032$ \\
\hline 0.8 & 390 & 800 & 200 & $-24.2 \pm 7.7$ & $-0.061 \pm 0.030$ \\
\hline 1 & 440 & 900 & 0 & 0 & 0 \\
\hline \multicolumn{6}{|c|}{$T=210.03 \mathrm{~K}$} \\
\hline 0 & 335 & 0 & 500 & 0 & 0 \\
\hline 0.2 & 405 & 100 & 400 & $-21.9 \pm 9.0$ & $-0.061 \pm 0.040$ \\
\hline 0.4 & 485 & 300 & 450 & $-33.8 \pm 8.7$ & $-0.099 \pm 0.039$ \\
\hline 0.5 & 525 & 350 & 350 & $-38.8 \pm 7.0$ & $-0.119 \pm 0.032$ \\
\hline 0.6 & 570 & 510 & 340 & $-40.0 \pm 7.2$ & $-0.126 \pm 0.033$ \\
\hline 0.7 & 615 & 630 & 270 & $-39.3 \pm 6.9$ & $-0.127 \pm 0.030$ \\
\hline 0.8 & 660 & 800 & 200 & $-30.5 \pm 6.2$ & $-0.100 \pm 0.028$ \\
\hline 1 & 750 & 900 & 0 & 0 & 0 \\
\hline \multicolumn{6}{|c|}{$T=220.14 \mathrm{~K}$} \\
\hline 0 & 495 & 0 & 500 & 0 & 0 \\
\hline 0.2 & 595 & 100 & 400 & $-24.6 \pm 8.2$ & $-0.081 \pm 0.041$ \\
\hline 0.4 & 700 & 300 & 450 & $-35.0 \pm 7.9$ & $-0.112 \pm 0.039$ \\
\hline 0.5 & 755 & 350 & 350 & $-38.8 \pm 8.3$ & $-0.122 \pm 0.040$ \\
\hline 0.6 & 810 & 510 & 340 & $-41.3 \pm 7.7$ & $-0.140 \pm 0.037$ \\
\hline 0.7 & 870 & 630 & 270 & $-37.3 \pm 7.0$ & $-0.122 \pm 0.033$ \\
\hline 0.8 & 930 & 800 & 200 & $-30.8 \pm 8.9$ & $-0.105 \pm 0.043$ \\
\hline 1 & 1050 & 900 & 0 & 0 & 0 \\
\hline
\end{tabular}


Table 3. Continued

\begin{tabular}{|c|c|c|c|c|c|}
\hline$x(\mathrm{Xe})$ & $p / \mathrm{kPa}$ & $N_{\mathrm{Xe}}$ & $N_{\text {ethane }}$ & $H_{\mathrm{m}}^{\mathrm{E}} / \mathrm{J} \cdot \mathrm{mol}^{-1}$ & $V_{\mathrm{m}}^{\mathrm{E}} / \mathrm{cm}^{3} \cdot \mathrm{mol}^{-1}$ \\
\hline \multicolumn{6}{|c|}{$T=230.05 \mathrm{~K}$} \\
\hline 0 & 700 & 0 & 500 & 0 & 0 \\
\hline 0.2 & 825 & 100 & 400 & $-22.3 \pm 9.9$ & $-0.085 \pm 0.055$ \\
\hline 0.4 & 960 & 300 & 450 & $-34.5 \pm 8.6$ & $-0.115 \pm 0.047$ \\
\hline 0.5 & 1030 & 350 & 350 & $-35.3 \pm 9.4$ & $-0.124 \pm 0.051$ \\
\hline 0.6 & 1100 & 510 & 340 & $-36.7 \pm 8.3$ & $-0.125 \pm 0.045$ \\
\hline 0.8 & 1255 & 800 & 200 & $-27.5 \pm 8.6$ & $-0.099 \pm 0.046$ \\
\hline 1 & 1415 & 900 & 0 & 0 & 0 \\
\hline \multicolumn{6}{|c|}{$T=239.3 \mathrm{~K}$} \\
\hline 0 & 950 & 0 & 500 & 0 & 0 \\
\hline 0.2 & 1095 & 100 & 400 & $-17.6 \pm 9.9$ & $-0.060 \pm 0.057$ \\
\hline 0.4 & 1260 & 300 & 450 & $-30.6 \pm 10.6$ & $-0.092 \pm 0.062$ \\
\hline 0.5 & 1345 & 350 & 350 & $-33.9 \pm 9.5$ & $-0.118 \pm 0.055$ \\
\hline 0.6 & 1435 & 510 & 340 & $-29.9 \pm 8.5$ & $-0.094 \pm 0.051$ \\
\hline 0.8 & 1630 & 800 & 200 & $-14.5 \pm 8.4$ & $-0.025 \pm 0.049$ \\
\hline 1 & 1835 & 900 & 0 & 0 & 0 \\
\hline \multicolumn{6}{|c|}{$T=250.17 \mathrm{~K}$} \\
\hline 0 & 1305 & 0 & 500 & 0 & 0 \\
\hline 0.2 & 1490 & 100 & 400 & $-25.3 \pm 11.0$ & $-0.100 \pm 0.075$ \\
\hline 0.4 & 1695 & 300 & 450 & $-35.3 \pm 9.7$ & $-0.140 \pm 0.065$ \\
\hline 0.5 & 1805 & 350 & 350 & $-35.9 \pm 9.1$ & $-0.130 \pm 0.061$ \\
\hline 0.6 & 1920 & 510 & 340 & $-33.9 \pm 9.9$ & $-0.123 \pm 0.065$ \\
\hline 0.8 & 2170 & 800 & 200 & $-21.9 \pm 8.4$ & $-0.074 \pm 0.054$ \\
\hline 1 & 2440 & 900 & 0 & 0 & 0 \\
\hline
\end{tabular}

potentials centered on the cabon atoms. This is a united atom force field, which means that $\mathrm{CH}_{n}$ groups are modeled as single pseudoatoms. Lennard-Jones parameters from the potential of Bohn et al. ${ }^{31}$ were used to model xenon. The parameters used in the calculations are presented in Tables 1 and 2, respectively, for nonbonded and bonded interactions. The cross interaction nonbonded parameters were obtained by Lorentz-Berthelot combining rules.

The calculations were carried out in the $N p T$ ensemble with a single box for a fixed pressure in each system slightly above the experimental (or estimated) vapor pressure of the mixture. A $15 \AA$ cutoff radius was used in the interaction calculations, and the neglect of long-range interactions beyond the cutoff radius was compensated by application of analytic tail corrections. Coulombic interactions were not considered, since all the molecules studied are neutral and apolar. For pure components, each system consisted of a total of 500 molecules for the $n$-alkane and 900 molecules for xenon with a box size chosen to obtain a density close to the experimental value. Mixtures were "prepared" by varying the proportions of molecules of each component, with the total number of molecules between 500 and 1000 molecules, observing the same criteria as above to fill the simulation box. The compositions (xenon mole fractions) studied for all cases were $0,0.2,0.4,0.5,0.6,0.8$, and 1 , and in the case of (xenon + propane), (xenon $+n$-butane), and (xenon + ethane), the latter for only two temperatures, also 0.7 , to better define the most asymmetric curves $\left(H_{\mathrm{m}}^{\mathrm{E}}\right.$ and $V_{\mathrm{m}}^{\mathrm{E}}$ as a function of composition). The simulations were performed for large temperature ranges (typically between 160 and $255 \mathrm{~K}$ ) at intervals of $\sim 10 \mathrm{~K}$. In some cases, temperatures for which experimental results of excess enthalpy or vapor pressures exist were chosen.
In each simulation, a preliminary equilibration run of 50000 $\mathrm{MC}$ cycles (each cycle consisting of a number of moves equal to the number of molecules in the system) was performed, followed by a production run for the calculation of averages, consisting of another 200000 cycles, which were divided into 20 blocks to calculate standard deviations. The Monte Carlo moves consisted of simulation box volume changes, coupled-decoupled configurational bias regrowths, translations of the center of mass, rotations about the center of mass, configurational bias molecule reinsertions in the simulation box and aggregation volume bias.

One thousand production configurations were used to obtain radial distribution functions for the three systems studied at the same temperature range, but only for pure components and equimolar mixtures Additional calculations were carried out for mixtures with xenon molar fractions of 0.2 and 0.8 at four different temperatures (161.40, 195.49, 225, and $245 \mathrm{~K}$ for (xenon + propane) and (xenon + $n$-butane) and 161.40, $195.49,220.14$, and 239.3 for (xenon + ethane)).

\section{RESULTS AND DISCUSSION}

Excess Functions. $H_{\mathrm{m}}^{\mathrm{E}}$ and excess molar volumes $V_{\mathrm{m}}^{\mathrm{E}}$ were obtained by simulation for mixtures of xenon with ethane, propane, and $n$-butane as a function of composition in a wide range of temperatures, typically between 161 and $255 \mathrm{~K}$. The results were fitted to Redlich-Kister equations of the form

$$
\frac{H_{\mathrm{m}}^{\mathrm{E}}}{R T x_{1} x_{2}}=A+B\left(x_{1}-x_{2}\right)+C\left(x_{1}-x_{2}\right)^{2}
$$


Table 4. Simulation Results of Excess Molar Enthalpy and Excess Molar Volume for Mixtures of Xenon with Propane As Function of Composition at Several Temperatures, Along System Pressure and Number of Molecules of Each Component for All the Simulations

\begin{tabular}{|c|c|c|c|c|c|}
\hline$x(\mathrm{Xe})$ & $p / \mathrm{kPa}$ & $N_{\text {Xe }}$ & $N_{\text {propane }}$ & $H_{\mathrm{m}}^{\mathrm{E}} / \mathrm{J} \cdot \mathrm{mol}^{-1}$ & $V_{\mathrm{m}}^{\mathrm{E}} / \mathrm{cm}^{3} \cdot \mathrm{mol}^{-1}$ \\
\hline \multicolumn{6}{|c|}{$T=161.40 \mathrm{~K}$} \\
\hline 0 & 5 & 0 & 500 & 0 & 0 \\
\hline 0.2 & 20 & 100 & 400 & $-17.3 \pm 9.0$ & $-0.055 \pm 0.025$ \\
\hline 0.4 & 35 & 300 & 450 & $-38.9 \pm 5.5$ & $-0.124 \pm 0.016$ \\
\hline 0.5 & 45 & 350 & 350 & $-44.5 \pm 8.1$ & $-0.142 \pm 0.023$ \\
\hline 0.6 & 55 & 510 & 340 & $-46.0 \pm 7.1$ & $-0.148 \pm 0.021$ \\
\hline 0.7 & 60 & 630 & 270 & $-46.1 \pm 6.8$ & $-0.146 \pm 0.020$ \\
\hline 0.8 & 70 & 800 & 200 & $-38.7 \pm 7.9$ & $-0.124 \pm 0.024$ \\
\hline 1 & 83 & 900 & 0 & 0 & 0 \\
\hline \multicolumn{6}{|c|}{$T=170 \mathrm{~K}$} \\
\hline 0 & 5 & 0 & 500 & 0 & 0 \\
\hline 0.2 & 30 & 100 & 400 & $-17.0 \pm 7.6$ & $-0.063 \pm 0.022$ \\
\hline 0.4 & 60 & 300 & 450 & $-36.3 \pm 6.7$ & $-0.130 \pm 0.020$ \\
\hline 0.5 & 70 & 350 & 350 & $-38.9 \pm 5.8$ & $-0.135 \pm 0.017$ \\
\hline 0.6 & 85 & 510 & 340 & $-43.9 \pm 7.6$ & $-0.153 \pm 0.023$ \\
\hline 0.7 & 100 & 630 & 270 & $-37.9 \pm 6.2$ & $-0.134 \pm 0.019$ \\
\hline 0.8 & 110 & 800 & 200 & $-34.0 \pm 6.3$ & $-0.117 \pm 0.019$ \\
\hline 1 & 137 & 900 & 0 & 0 & 0 \\
\hline \multicolumn{6}{|c|}{$T=182.34 \mathrm{~K}$} \\
\hline 0 & 10 & 0 & 500 & 0 & 0 \\
\hline 0.2 & 55 & 100 & 400 & $-21.1 \pm 6.7$ & $-0.086 \pm 0.021$ \\
\hline 0.4 & 100 & 300 & 450 & $-36.9 \pm 5.7$ & $-0.152 \pm 0.018$ \\
\hline 0.5 & 125 & 350 & 350 & $-41.9 \pm 7.9$ & $-0.176 \pm 0.026$ \\
\hline 0.6 & 150 & 510 & 340 & $-44.7 \pm 6.9$ & $-0.184 \pm 0.023$ \\
\hline 0.7 & 175 & 630 & 270 & $-38.3 \pm 7.3$ & $-0.160 \pm 0.025$ \\
\hline 0.8 & 200 & 800 & 200 & $-30.8 \pm 7.4$ & $-0.134 \pm 0.025$ \\
\hline 1 & 250 & 900 & 0 & 0 & 0 \\
\hline \multicolumn{6}{|c|}{$T=195.49 \mathrm{~K}$} \\
\hline 0 & 15 & 0 & 500 & 0 & 0 \\
\hline 0.2 & 90 & 100 & 400 & $-28.5 \pm 7.3$ & $-0.127 \pm 0.028$ \\
\hline 0.4 & 170 & 300 & 450 & $-45.0 \pm 7.5$ & $-0.207 \pm 0.029$ \\
\hline 0.5 & 215 & 350 & 350 & $-47.0 \pm 7.8$ & $-0.224 \pm 0.030$ \\
\hline 0.6 & 255 & 510 & 340 & $-50.5 \pm 6.4$ & $-0.241 \pm 0.025$ \\
\hline 0.7 & 300 & 630 & 270 & $-48.1 \pm 7.5$ & $-0.226 \pm 0.029$ \\
\hline 0.8 & 350 & 800 & 200 & $-36.8 \pm 7.5$ & $-0.179 \pm 0.029$ \\
\hline 1 & 440 & 900 & 0 & 0 & 0 \\
\hline \multicolumn{6}{|c|}{$T=205 \mathrm{~K}$} \\
\hline 0 & 30 & 0 & 500 & 0 & 0 \\
\hline 0.2 & 145 & 100 & 400 & $-29.8 \pm 8.4$ & $-0.157 \pm 0.031$ \\
\hline 0.4 & 270 & 300 & 450 & $-54.0 \pm 9.1$ & $-0.281 \pm 0.034$ \\
\hline 0.5 & 330 & 350 & 350 & $-60.2 \pm 8.1$ & $-0.317 \pm 0.031$ \\
\hline 0.6 & 390 & 510 & 340 & $-58.2 \pm 7.8$ & $-0.313 \pm 0.030$ \\
\hline 0.7 & 450 & 630 & 270 & $-56.3 \pm 6.4$ & $-0.302 \pm 0.025$ \\
\hline 0.8 & 510 & 800 & 200 & $-48.9 \pm 7.4$ & $-0.262 \pm 0.030$ \\
\hline 1 & 630 & 900 & 0 & 0 & 0 \\
\hline \multicolumn{6}{|c|}{$T=215 \mathrm{~K}$} \\
\hline 0 & 50 & 0 & 500 & 0 & 0 \\
\hline 0.2 & 220 & 100 & 400 & $-36.2 \pm 9.0$ & $-0.207 \pm 0.017$ \\
\hline 0.4 & 385 & 300 & 450 & $-54.9 \pm 8.7$ & $-0.331 \pm 0.024$ \\
\hline 0.5 & 470 & 350 & 350 & $-62.9 \pm 7.7$ & $-0.368 \pm 0.023$ \\
\hline
\end{tabular}


Table 4. Continued

\begin{tabular}{|c|c|c|c|c|c|}
\hline$x(\mathrm{Xe})$ & $p / \mathrm{kPa}$ & $N_{\mathrm{Xe}}$ & $N_{\text {propane }}$ & $H_{\mathrm{m}}^{\mathrm{E}} / \mathrm{J} \cdot \mathrm{mol}^{-1}$ & $V_{\mathrm{m}}^{\mathrm{E}} / \mathrm{cm}^{3} \cdot \mathrm{mol}^{-1}$ \\
\hline 0.6 & 555 & 510 & 340 & $-61.8 \pm 8.5$ & $-0.380 \pm 0.029$ \\
\hline 0.7 & 640 & 630 & 270 & $-54.6 \pm 7.4$ & $-0.342 \pm 0.027$ \\
\hline 0.8 & 725 & 800 & 200 & $-47.2 \pm 7.6$ & $-0.294 \pm 0.030$ \\
\hline 1 & 890 & 900 & 0 & 0 & 0 \\
\hline \multicolumn{6}{|c|}{$T=225 \mathrm{~K}$} \\
\hline 0 & 80 & 0 & 500 & 0 & 0 \\
\hline 0.2 & 310 & 100 & 400 & $-35.8 \pm 8.7$ & $-0.246 \pm 0.037$ \\
\hline 0.4 & 535 & 300 & 450 & $-71.0 \pm 7.2$ & $-0.454 \pm 0.031$ \\
\hline 0.5 & 650 & 350 & 350 & $-76.5 \pm 7.2$ & $-0.499 \pm 0.032$ \\
\hline 0.6 & 765 & 510 & 340 & $-84.3 \pm 7.3$ & $-0.539 \pm 0.033$ \\
\hline 0.7 & 880 & 630 & 270 & $-72.5 \pm 7.6$ & $-0.480 \pm 0.034$ \\
\hline 0.8 & 995 & 800 & 200 & $-57.5 \pm 7.2$ & $-0.389 \pm 0.034$ \\
\hline 1 & 1225 & 900 & 0 & 0 & 0 \\
\hline \multicolumn{6}{|c|}{$T=235 \mathrm{~K}$} \\
\hline 0 & 125 & 0 & 500 & 0 & 0 \\
\hline 0.2 & 425 & 100 & 400 & $-47.8 \pm 10.7$ & $-0.338 \pm 0.046$ \\
\hline 0.4 & 730 & 300 & 450 & $-77.8 \pm 8.5$ & $-0.564 \pm 0.039$ \\
\hline 0.5 & 880 & 350 & 350 & $-86.5 \pm 7.8$ & $-0.639 \pm 0.037$ \\
\hline 0.6 & 1030 & 510 & 340 & $-89.0 \pm 8.4$ & $-0.652 \pm 0.041$ \\
\hline 0.7 & 1180 & 630 & 270 & $-79.2 \pm 7.0$ & $-0.601 \pm 0.035$ \\
\hline 0.8 & 1335 & 800 & 200 & $-60.0 \pm 7.7$ & $-0.472 \pm 0.039$ \\
\hline 1 & 1635 & 900 & 0 & 0 & 0 \\
\hline \multicolumn{6}{|c|}{$T=245 \mathrm{~K}$} \\
\hline 0 & 185 & 0 & 500 & 0 & 0 \\
\hline 0.2 & 575 & 100 & 400 & $-45.4 \pm 10.0$ & $-0.398 \pm 0.045$ \\
\hline 0.4 & 965 & 300 & 450 & $-87.5 \pm 7.8$ & $-0.729 \pm 0.038$ \\
\hline 0.5 & 1160 & 350 & 350 & $-100.4 \pm 8.7$ & $-0.832 \pm 0.043$ \\
\hline 0.6 & 1355 & 510 & 340 & $-105.8 \pm 7.6$ & $-0.876 \pm 0.039$ \\
\hline 0.7 & 1550 & 630 & 270 & $-102.5 \pm 8.4$ & $-0.841 \pm 0.044$ \\
\hline 0.8 & 1745 & 800 & 200 & $-83.3 \pm 8.1$ & $-0.698 \pm 0.045$ \\
\hline 1 & 2140 & 900 & 0 & 0 & 0 \\
\hline \multicolumn{6}{|c|}{$T=255 \mathrm{~K}$} \\
\hline 0 & 265 & 0 & 500 & 0 & 0 \\
\hline 0.2 & 760 & 100 & 400 & $-67.3 \pm 9.8$ & $-0.597 \pm 0.050$ \\
\hline 0.4 & 1255 & 300 & 450 & $-113.2 \pm 8.6$ & $-1.014 \pm 0.046$ \\
\hline 0.5 & 1505 & 350 & 350 & $-125.3 \pm 10.0$ & $-1.132 \pm 0.056$ \\
\hline 0.6 & 1750 & 510 & 340 & $-132.4 \pm 9.1$ & $-1.198 \pm 0.052$ \\
\hline 0.7 & 2000 & 630 & 270 & $-128.2 \pm 8.8$ & $-1.168 \pm 0.054$ \\
\hline 0.8 & 2250 & 800 & 200 & $-105.2 \pm 8.7$ & $-0.977 \pm 0.054$ \\
\hline 1 & 2740 & 900 & 0 & 0 & 0 \\
\hline
\end{tabular}

for excess molar enthalpies and

$$
\frac{V_{\mathrm{m}}^{\mathrm{E}}}{x_{1} x_{2}}=D+E\left(x_{1}-x_{2}\right)+F\left(x_{1}-x_{2}\right)^{2}
$$

for excess molar volumes. The simulation results are compiled in Table 3 for (xenon + ethane), Table 4 for (xenon + propane), and Table 5 for (xenon $+n$-butane), and the corresponding Redlish-Kister fitting coefficients can be found in Tables $6\left(H_{\mathrm{m}}^{\mathrm{E}}\right)$ and $7\left(V_{\mathrm{m}}^{\mathrm{E}}\right)$.

For (xenon + ethane), the simulated excess enthalpies and excess volumes are plotted as a function of composition in Figure 1a and Figure 1c, at each temperature, and as a function of temperature in Figure $1 \mathrm{~b}$ and Figure 1d, at each composition.
Experimental excess volumes and excess enthalpies obtained in our group at a single temperature $\left(161.40 \mathrm{~K}\right.$ for $V_{\mathrm{m}}^{\mathrm{E}}$ and $163.0 \mathrm{~K}$ for $\left.H_{\mathrm{m}}^{\mathrm{E}}\right)^{23}$ are also included in Figure 1a,c. As can be seen, the simulation results for both properties are systematically less negatives than the experimental ones, but the sign and the order of magnitude are predicted, and the overall agreement can be considered very good. It should be emphasized that excess properties are difficult quantities to obtain either experimentally or by simulation. Consequently, large relative uncertainties are common, and their prediction is a considerable challenge to both theory and simulation.

It can also be observed that the excess molar enthalpy for mixtures of (xenon + ethane) is almost independent of temperature 
Table 5. Simulation Results of Excess Molar Enthalpy and Excess Molar Volume for Mixtures of Xenon with $\boldsymbol{n}$-Butane as a Function of Composition at Several Temperatures, along with System Pressure and Number of Molecules of Each Component for All the Simulations

\begin{tabular}{|c|c|c|c|c|c|}
\hline$x(\mathrm{Xe})$ & $p / \mathrm{kPa}$ & $N_{\mathrm{Xe}}$ & $N_{\text {butane }}$ & $H_{\mathrm{m}}^{\mathrm{E}} / \mathrm{J} \cdot \mathrm{mol}^{-1}$ & $V_{\mathrm{m}}^{\mathrm{E}} / \mathrm{cm}^{3} \cdot \mathrm{mol}^{-1}$ \\
\hline \multicolumn{6}{|c|}{$T=161.40 \mathrm{~K}$} \\
\hline 0 & 0.1 & 0 & 500 & 0 & 0 \\
\hline 0.2 & 20 & 100 & 400 & $-40.5 \pm 14.4$ & $-0.162 \pm 0.037$ \\
\hline 0.4 & 35 & 300 & 450 & $-63.9 \pm 10.7$ & $-0.265 \pm 0.029$ \\
\hline 0.5 & 46 & 350 & 350 & $-71.1 \pm 10.0$ & $-0.299 \pm 0.027$ \\
\hline 0.6 & 50 & 510 & 340 & $-68.9 \pm 9.7$ & $-0.299 \pm 0.027$ \\
\hline 0.7 & 60 & 630 & 270 & $-65.4 \pm 9.3$ & $-0.282 \pm 0.027$ \\
\hline 0.8 & 70 & 800 & 200 & $-54.5 \pm 8.5$ & $-0.240 \pm 0.024$ \\
\hline 1 & 85 & 900 & 0 & 0 & 0 \\
\hline \multicolumn{6}{|c|}{$T=170 \mathrm{~K}$} \\
\hline 0 & 0.5 & 0 & 500 & 0 & 0 \\
\hline 0.2 & 30 & 100 & 400 & $-38.5 \pm 13.4$ & $-0.166 \pm 0.031$ \\
\hline 0.4 & 55 & 300 & 450 & $-63.9 \pm 10.9$ & $-0.289 \pm 0.026$ \\
\hline 0.5 & 70 & 350 & 350 & $-63.8 \pm 10.2$ & $-0.317 \pm 0.026$ \\
\hline 0.6 & 85 & 510 & 340 & $-62.7 \pm 9.3$ & $-0.311 \pm 0.025$ \\
\hline 0.7 & 95 & 630 & 270 & $-65.4 \pm 7.8$ & $-0.318 \pm 0.022$ \\
\hline 0.8 & 110 & 800 & 200 & $-49.8 \pm 8.9$ & $-0.255 \pm 0.027$ \\
\hline 1 & 140 & 900 & 0 & 0 & 0 \\
\hline \multicolumn{6}{|c|}{$T=182.34 \mathrm{~K}$} \\
\hline 0 & 1 & 0 & 500 & 0 & 0 \\
\hline 0.2 & 45 & 100 & 400 & $-43.1 \pm 13.6$ & $-0.214 \pm 0.034$ \\
\hline 0.4 & 95 & 300 & 450 & $-65.4 \pm 11.1$ & $-0.346 \pm 0.029$ \\
\hline 0.5 & 120 & 350 & 350 & $-71.7 \pm 10.6$ & $-0.393 \pm 0.028$ \\
\hline 0.6 & 145 & 510 & 340 & $-72.1 \pm 9.4$ & $-0.402 \pm 0.028$ \\
\hline 0.7 & 170 & 630 & 270 & $-63.9 \pm 8.7$ & $-0.379 \pm 0.026$ \\
\hline 0.8 & 195 & 800 & 200 & $-49.8 \pm 9.2$ & $-0.303 \pm 0.030$ \\
\hline 1 & 250 & 900 & 0 & 0 & 0 \\
\hline \multicolumn{6}{|c|}{$T=195.49 \mathrm{~K}$} \\
\hline 0 & 2 & 0 & 500 & 0 & 0 \\
\hline 0.2 & 80 & 100 & 400 & $-39.3 \pm 9.9$ & $-0.258 \pm 0.031$ \\
\hline 0.4 & 160 & 300 & 450 & $-79.0 \pm 7.9$ & $-0.457 \pm 0.025$ \\
\hline 0.5 & 205 & 350 & 350 & $-87.7 \pm 7.6$ & $-0.525 \pm 0.025$ \\
\hline 0.6 & 250 & 510 & 340 & $-92.9 \pm 6.6$ & $-0.554 \pm 0.024$ \\
\hline 0.7 & 295 & 630 & 270 & $-80.9 \pm 6.8$ & $-0.504 \pm 0.024$ \\
\hline 0.8 & 340 & 800 & 200 & $-68.8 \pm 6.9$ & $-0.435 \pm 0.025$ \\
\hline 1 & 440 & 900 & 0 & 0 & 0 \\
\hline \multicolumn{6}{|c|}{$T=205 \mathrm{~K}$} \\
\hline 0 & 5 & 0 & 500 & 0 & 0 \\
\hline 0.2 & 130 & 100 & 400 & $-46.4 \pm 12.6$ & $-0.319 \pm 0.038$ \\
\hline 0.4 & 260 & 300 & 450 & $-87.7 \pm 10.1$ & $-0.564 \pm 0.032$ \\
\hline 0.5 & 320 & 350 & 350 & $-94.0 \pm 8.7$ & $-0.619 \pm 0.028$ \\
\hline 0.6 & 380 & 510 & 340 & $-96.0 \pm 9.4$ & $-0.647 \pm 0.034$ \\
\hline 0.7 & 445 & 630 & 270 & $-88.1 \pm 7.9$ & $-0.612 \pm 0.029$ \\
\hline 0.8 & 505 & 800 & 200 & $-76.9 \pm 6.7$ & $-0.534 \pm 0.026$ \\
\hline 1 & 630 & 900 & 0 & 0 & 0 \\
\hline \multicolumn{6}{|c|}{$T=215 \mathrm{~K}$} \\
\hline 0 & 7 & 0 & 500 & 0 & 0 \\
\hline 0.2 & 185 & 100 & 400 & $-45.5 \pm 12.7$ & $-0.364 \pm 0.039$ \\
\hline 0.4 & 360 & 300 & 450 & $-90.9 \pm 8.9$ & $-0.656 \pm 0.031$ \\
\hline
\end{tabular}


Table 5. Continued

\begin{tabular}{|c|c|c|c|c|c|}
\hline$x(\mathrm{Xe})$ & $p / \mathrm{kPa}$ & $N_{\mathrm{Xe}}$ & $N_{\text {butane }}$ & $H_{\mathrm{m}}^{\mathrm{E}} / \mathrm{J} \cdot \mathrm{mol}^{-1}$ & $V_{\mathrm{m}}^{\mathrm{E}} / \mathrm{cm}^{3} \cdot \mathrm{mol}^{-1}$ \\
\hline 0.5 & 450 & 350 & 350 & $-99.4 \pm 10.6$ & $-0.742 \pm 0.038$ \\
\hline 0.6 & 540 & 510 & 340 & $-102.5 \pm 8.4$ & $-0.781 \pm 0.030$ \\
\hline 0.7 & 625 & 630 & 270 & $-100.3 \pm 9.0$ & $-0.759 \pm 0.035$ \\
\hline 0.8 & 715 & 800 & 200 & $-88.3 \pm 8.1$ & $-0.665 \pm 0.033$ \\
\hline 1 & 890 & 900 & 0 & 0 & 0 \\
\hline \multicolumn{6}{|c|}{$T=225 \mathrm{~K}$} \\
\hline 0 & 15 & 0 & 500 & 0 & 0 \\
\hline 0.2 & 255 & 100 & 400 & $-55.7 \pm 11.3$ & $-0.442 \pm 0.040$ \\
\hline 0.4 & 495 & 300 & 450 & $-103.2 \pm 10.4$ & $-0.817 \pm 0.037$ \\
\hline 0.5 & 620 & 350 & 350 & $-113.8 \pm 9.4$ & $-0.915 \pm 0.036$ \\
\hline 0.6 & 740 & 510 & 340 & $-126.5 \pm 7.8$ & $-0.991 \pm 0.031$ \\
\hline 0.7 & 860 & 630 & 270 & $-122.6 \pm 9.3$ & $-0.965 \pm 0.040$ \\
\hline 0.8 & 980 & 800 & 200 & $-104.2 \pm 8.9$ & $-0.839 \pm 0.039$ \\
\hline 1 & 1225 & 900 & 0 & 0 & 0 \\
\hline \multicolumn{6}{|c|}{$T=235 \mathrm{~K}$} \\
\hline 0 & 20 & 0 & 500 & 0 & 0 \\
\hline 0.2 & 345 & 100 & 400 & $-64.0 \pm 11.0$ & $-0.566 \pm 0.038$ \\
\hline 0.4 & 665 & 300 & 450 & $-108.7 \pm 9.9$ & $-0.990 \pm 0.033$ \\
\hline 0.5 & 830 & 350 & 350 & $-131.9 \pm 11.5$ & $-1.159 \pm 0.043$ \\
\hline 0.6 & 990 & 510 & 340 & $-146.4 \pm 9.0$ & $-1.259 \pm 0.033$ \\
\hline 0.7 & 1150 & 630 & 270 & $-142.6 \pm 8.1$ & $-1.236 \pm 0.031$ \\
\hline 0.8 & 1315 & 800 & 200 & $-128.3 \pm 9.1$ & $-1.108 \pm 0.038$ \\
\hline 1 & 1635 & 900 & 0 & 0 & 0 \\
\hline \multicolumn{6}{|c|}{$T=245 \mathrm{~K}$} \\
\hline 0 & 35 & 0 & 500 & 0 & 0 \\
\hline 0.2 & 455 & 100 & 400 & $-76.1 \pm 11.7$ & $-0.737 \pm 0.045$ \\
\hline 0.4 & 875 & 360 & 540 & $-141.8 \pm 11.0$ & $-1.312 \pm 0.045$ \\
\hline 0.5 & 1085 & 450 & 450 & $-167.6 \pm 9.9$ & $-1.533 \pm 0.043$ \\
\hline 0.6 & 1295 & 600 & 400 & $-180.3 \pm 9.9$ & $-1.644 \pm 0.046$ \\
\hline 0.7 & 1505 & 630 & 270 & $-172.6 \pm 11.1$ & $-1.614 \pm 0.053$ \\
\hline 0.8 & 1720 & 800 & 200 & $-149.2 \pm 9.9$ & $-1.417 \pm 0.052$ \\
\hline 1 & 2140 & 900 & 0 & 0 & 0 \\
\hline \multicolumn{6}{|c|}{$T=255 \mathrm{~K}$} \\
\hline 0 & 50 & 0 & 500 & 0 & 0 \\
\hline 0.2 & 590 & 100 & 400 & $-104.7 \pm 12.1$ & $-0.990 \pm 0.047$ \\
\hline 0.4 & 1130 & 360 & 540 & $-171.3 \pm 10.3$ & $-1.716 \pm 0.046$ \\
\hline 0.5 & 1395 & 450 & 450 & $-209.6 \pm 10.1$ & $-2.042 \pm 0.047$ \\
\hline 0.6 & 1665 & 600 & 400 & $-232.6 \pm 9.3$ & $-2.242 \pm 0.045$ \\
\hline 0.7 & 1935 & 630 & 270 & $-221.4 \pm 9.2$ & $-2.193 \pm 0.050$ \\
\hline 0.8 & 2205 & 800 & 200 & $-195.0 \pm 9.8$ & $-1.963 \pm 0.057$ \\
\hline 1 & 2740 & 900 & 0 & 0 & 0 \\
\hline
\end{tabular}

in the studied range (almost $90 \mathrm{~K}$ ), whereas a slight decrease with increasing temperature is observed for excess molar volume data. Nunes da Ponte et al. ${ }^{32}$ obtained excess Gibbs energies from VLE experimental data for (xenon + ethane) mixtures at temperatures between 210 and $284 \mathrm{~K}$, from which they estimated excess molar enthalpies for equimolar mixtures as a function of temperature. In Figure 1e, our simulation results are compared with those estimated by Nunes da Ponte et al. As can be seen, the agreement is remarkable in the low temperature limit, but deviations are found at higher temperatures, since the excess enthalpies estimated by Nunes da Ponte et al. decrease with the increasing temperature. Again, given the uncertainty of the simulation and experimental results, the overall agreement can be considered quite good. Also included in Figure 1e is the experimental data point from ref 23. Both the simulation results and those of Nunes da Ponte et al. underestimate this result by $\sim 30 \%$.

The simulation results for (xenon + propane) and (xenon + $n$-butane) are shown in Figures 2 and 3, respectively. The only results available for comparison are indirect estimations of equimolar excess enthalpy and experimental excess molar volumes at $161.40 \mathrm{~K}$ for (xenon + propane) ${ }^{23}$ and at $182.34 \mathrm{~K}$ for (xenon $+n$-butane), ${ }^{24}$ which are also included in the figures. For these two latter systems, the temperature dependence of the magnitude of both excess functions is much more apparent. As in 
Table 6. Parameters of the Redlich-Kister Fittings for Excess Molar Enthalpies

\begin{tabular}{|c|c|c|c|}
\hline$T / \mathrm{K}$ & $A$ & $B$ & $C$ \\
\hline \multicolumn{4}{|c|}{ (Xenon + Ethane) } \\
\hline 161.4 & $-0.1061 \pm 0.0006$ & $0.009 \pm 0.001$ & $-0.004 \pm 0.003$ \\
\hline 170 & $-0.098 \pm 0.002$ & $0.010 \pm 0.004$ & $0.003 \pm 0.01$ \\
\hline 182.34 & $-0.087 \pm 0.002$ & $0.008 \pm 0.004$ & $-0.01 \pm 0.01$ \\
\hline 195.49 & $-0.082 \pm 0.002$ & $0.011 \pm 0.004$ & $-0.011 \pm 0.009$ \\
\hline 210.03 & $-0.089 \pm 0.002$ & $0.029 \pm 0.004$ & $-0.016 \pm 0.009$ \\
\hline 220.14 & $-0.085 \pm 0.002$ & $0.019 \pm 0.003$ & $-0.026 \pm 0.008$ \\
\hline 230.05 & $-0.076 \pm 0.001$ & $0.014 \pm 0.002$ & $-0.016 \pm 0.005$ \\
\hline 239.3 & $-0.066 \pm 0.001$ & $-0.007 \pm 0.002$ & $0.043 \pm 0.005$ \\
\hline 250.17 & $-0.0685 \pm 0.0006$ & $-0.008 \pm 0.001$ & $-0.007 \pm 0.003$ \\
\hline \multicolumn{4}{|c|}{ (Xenon + Propane) } \\
\hline 161.4 & $-0.132 \pm 0.003$ & $0.080 \pm 0.005$ & $0.005 \pm 0.01$ \\
\hline 170 & $-0.114 \pm 0.004$ & $0.058 \pm 0.007$ & $0.006 \pm 0.02$ \\
\hline 182.34 & $-0.111 \pm 0.002$ & $0.034 \pm 0.004$ & $0.01 \pm 0.01$ \\
\hline 195.49 & $-0.121 \pm 0.003$ & $0.030 \pm 0.005$ & $-0.02 \pm 0.01$ \\
\hline 205 & $-0.137 \pm 0.004$ & $0.053 \pm 0.006$ & $-0.02 \pm 0.02$ \\
\hline 215 & $-0.136 \pm 0.003$ & $0.030 \pm 0.005$ & $-0.02 \pm 0.01$ \\
\hline 225 & $-0.170 \pm 0.004$ & $0.060 \pm 0.006$ & $0.04 \pm 0.02$ \\
\hline 235 & $-0.178 \pm 0.003$ & $0.036 \pm 0.005$ & $0.02 \pm 0.01$ \\
\hline 245 & $-0.198 \pm 0.001$ & $0.098 \pm 0.002$ & $0.00003 \pm 0.005$ \\
\hline 255 & $-0.239 \pm 0.002$ & $0.095 \pm 0.003$ & $-0.044 \pm 0.008$ \\
\hline \multicolumn{4}{|c|}{ (Xenon $+n$-Butane $)$} \\
\hline 161.4 & $-0.207 \pm 0.003$ & $0.098 \pm 0.002$ & $0.00003 \pm 0.005$ \\
\hline 170 & $-0.186 \pm 0.007$ & $0.04 \pm 0.01$ & $-0.03 \pm 0.03$ \\
\hline 182.34 & $-0.189 \pm 0.002$ & $0.026 \pm 0.004$ & $-0.007 \pm 0.01$ \\
\hline 195.49 & $-0.217 \pm 0.005$ & $0.089 \pm 0.008$ & $0.03 \pm 0.02$ \\
\hline 205 & $-0.221 \pm 0.006$ & $0.08 \pm 0.01$ & $-0.01 \pm 0.03$ \\
\hline 215 & $-0.222 \pm 0.006$ & $0.12 \pm 0.01$ & $-0.03 \pm 0.03$ \\
\hline 225 & $-0.250 \pm 0.003$ & $0.135 \pm 0.005$ & $-0.05 \pm 0.01$ \\
\hline 235 & $-0.267 \pm 0.004$ & $0.172 \pm 0.006$ & $-0.11 \pm 0.02$ \\
\hline 245 & $-0.327 \pm 0.002$ & $0.185 \pm 0.004$ & $-0.05 \pm 0.01$ \\
\hline 255 & $-0.392 \pm 0.008$ & $0.23 \pm 0.01$ & $-0.14 \pm 0.04$ \\
\hline
\end{tabular}

the case of (xenon + ethane), the simulation results reproduce the correct sign and order of magnitude of the excess volumes, but underestimate the experimental values by $\sim 50 \%$. For the excess enthalpies, the simulations predict negative values at all temperatures and compositions, but those estimated from the temperature dependence of the experimental Gibbs energies are positive $\left(H_{\mathrm{m}}^{\mathrm{E}}(x=0.5)=+138 \pm 36 \mathrm{~J} \cdot \mathrm{mol}^{-1}\right.$ for (xenon + propane) and $H_{\mathrm{m}}^{\mathrm{E}}(x=0.5)=+17 \pm 70 \mathrm{~J} \cdot \mathrm{mol}^{-1}$ for $($ xenon $+n$ butane)). However, given the small value of $H_{\mathrm{m}}^{\mathrm{E}}$ for (xenon $+n$ butane) and the large uncertainty, the possibility of $H_{\mathrm{m}}^{\mathrm{E}}$ being small and negative for this system cannot be excluded.

Figures 2 and 3 also show that for (xenon + propane) and (xenon $+n$-butane), both excess functions decrease with temperature. However, unlike for mixtures of $n$-alkanes, the trend is not linear. Both properties remain nearly constant at the lowest temperatures, then start to decrease with increasing temperature, the curve becoming steeper as the highest temperatures are reached. Interestingly, this is the trend reported by Nunes da Ponte et al. for $H_{\mathrm{m}}^{\mathrm{E}}(x=0.5)$ and $V_{\mathrm{m}}^{\mathrm{E}}(x=0.5)$ as a function of the temperature of (xenon + ethane), as predicted by the
Table 7. Parameters of the Redlich-Kister Fittings for Excess Molar Volumes

\begin{tabular}{lllc}
$T / \mathrm{K}$ & \multicolumn{1}{c}{$D$} & \multicolumn{1}{c}{$E$} & $F$ \\
\multicolumn{4}{c}{ (Xenon + Ethane) } \\
161.4 & $-0.2640 \pm 0.0006$ & $0.028 \pm 0.001$ & $-0.022 \pm 0.003$ \\
170 & $-0.275 \pm 0.006$ & $0.02 \pm 0.01$ & $0.03 \pm 0.03$ \\
182.34 & $-0.27 \pm 0.01$ & $0.03 \pm 0.03$ & $-0.07 \pm 0.06$ \\
195.49 & $-0.34 \pm 0.02$ & $0.07 \pm 0.03$ & $-0.01 \pm 0.08$ \\
210.03 & $-0.47 \pm 0.01$ & $0.23 \pm 0.02$ & $-0.09 \pm 0.06$ \\
220.14 & $-0.50 \pm 0.02$ & $0.14 \pm 0.03$ & $-0.21 \pm 0.08$ \\
230.05 & $-0.492 \pm 0.004$ & $0.076 \pm 0.007$ & $-0.23 \pm 0.02$ \\
239.3 & $-0.43 \pm 0.03$ & $-0.16 \pm 0.06$ & $0.5 \pm 0.2$ \\
250.17 & $-0.54 \pm 0.01$ & $-0.14 \pm 0.02$ & $-0.03 \pm 0.05$
\end{tabular}

$\begin{array}{ll}T / \mathrm{K} & D \\ 161.4 & -0.57 \pm 0.01 \\ 170 & -0.57 \pm 0.02 \\ 182.34 & -0.70 \pm 0.01 \\ 195.49 & -0.92 \pm 0.01 \\ 205 & -1.24 \pm 0.03 \\ 215 & -1.46 \pm 0.02 \\ 225 & -2.04 \pm 0.03 \\ 235 & -2.55 \pm 0.02 \\ 245 & -3.332 \pm 0.003 \\ 255 & -4.56 \pm 0.02\end{array}$

(Xenon + Propane $)$

$\begin{array}{ll}E & F \\ 0.34 \pm 0.02 & 0.03 \pm 0.05 \\ 0.26 \pm 0.03 & 0.03 \pm 0.09 \\ 0.24 \pm 0.02 & 0.05 \pm 0.06 \\ 0.29 \pm 0.03 & -0.12 \pm 0.07 \\ 0.51 \pm 0.04 & -0.2 \pm 0.1 \\ 0.43 \pm 0.04 & -0.26 \pm 0.09 \\ 0.75 \pm 0.05 & 0.2 \pm 0.1 \\ 0.73 \pm 0.04 & 0.03 \pm 0.1 \\ 1.562 \pm 0.006 & -0.26 \pm 0.01 \\ 2.00 \pm 0.04 & -1.02 \pm 0.09\end{array}$

$($ Xenon $+n$-Butane $)$

\begin{tabular}{llll}
$T / \mathrm{K}$ & $D$ & $E$ & $F$ \\
161.4 & $-1.18 \pm 0.01$ & $0.40 \pm 0.02$ & $-0.21 \pm 0.05$ \\
170 & $-1.26 \pm 0.03$ & $0.46 \pm 0.05$ & $-0.2 \pm 0.1$ \\
182.34 & $-1.56 \pm 0.02$ & $0.48 \pm 0.03$ & $-0.15 \pm 0.07$ \\
195.49 & $-2.08 \pm 0.03$ & $0.89 \pm 0.05$ & $-0.2 \pm 0.1$ \\
205 & $-2.48 \pm 0.04$ & $1.06 \pm 0.06$ & $-0.5 \pm 0.2$ \\
215 & $-2.95 \pm 0.03$ & $1.51 \pm 0.06$ & $-0.7 \pm 0.2$ \\
225 & $-3.70 \pm 0.04$ & $2.02 \pm 0.06$ & $-0.8 \pm 0.2$ \\
235 & $-4.60 \pm 0.04$ & $2.77 \pm 0.07$ & $-1.7 \pm 0.2$ \\
245 & $-6.09 \pm 0.04$ & $3.49 \pm 0.06$ & $-1.7 \pm 0.2$ \\
255 & $-8.11 \pm 0.08$ & $5.0 \pm 0.1$ & $-3.0 \pm 0.3$ \\
\hline
\end{tabular}

Gubbins et al. ${ }^{33}$ version of perturbation theory. It is also worth noting that an extrapolation of the high temperature regime of the $H_{\mathrm{m}}^{\mathrm{E}}$ curves for both systems may lead to small and positive values of $H_{\mathrm{m}}^{\mathrm{E}}$ at the lowest temperatures, which are much closer to the experimental estimations. Vega ${ }^{34}$ and Blas ${ }^{22,35}$ have shown that for mixtures of $n$-alkanes, the experimentally observed positive values of $H_{\mathrm{m}}^{\mathrm{E}}$ at low temperatures were due to conformational changes in the molecules when they are mixed. Although conformational changes are included in the TraPPE model used in the present simulations, it is nevertheless a united atom model. It is possible that a more detailed, fully atomistic force field is necessary to deal with conformations in a more realistic way and account for the differences in $H_{\mathrm{m}}^{\mathrm{E}}$ at low temperature. In addition, given the size of the xenon atom, the use of a polarizable model might also be valuable. This will be the object of future work.

Following the procedure previously described for mixtures of $n$-alkanes, we have obtained the temperature at which $H_{\mathrm{m}}^{\mathrm{E}}$ and $V_{\mathrm{m}}^{\mathrm{E}}$ vanish for the three (xenon $+n$-alkane) systems at all the 


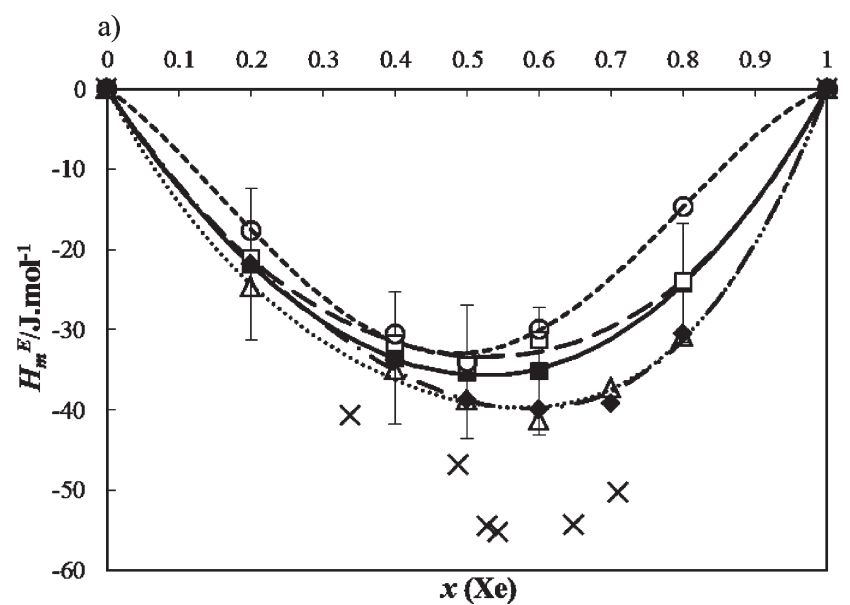

b)

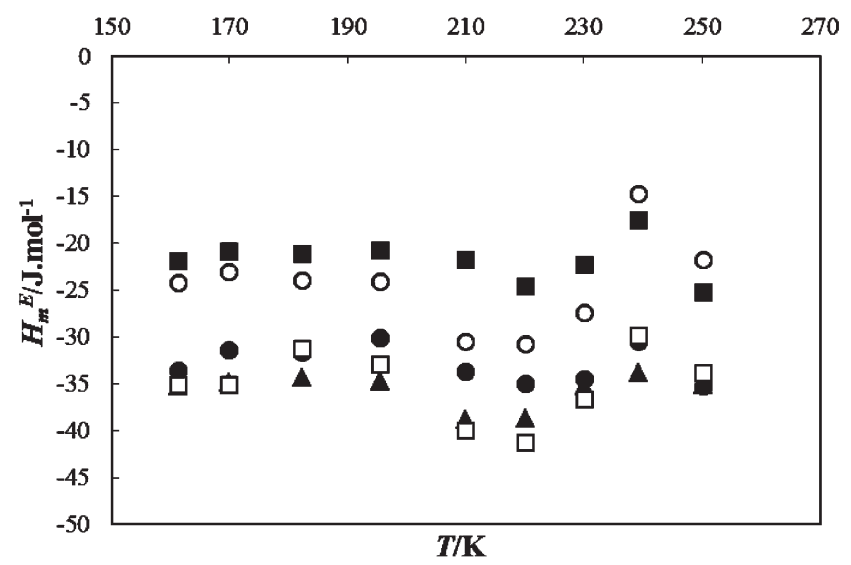

c)

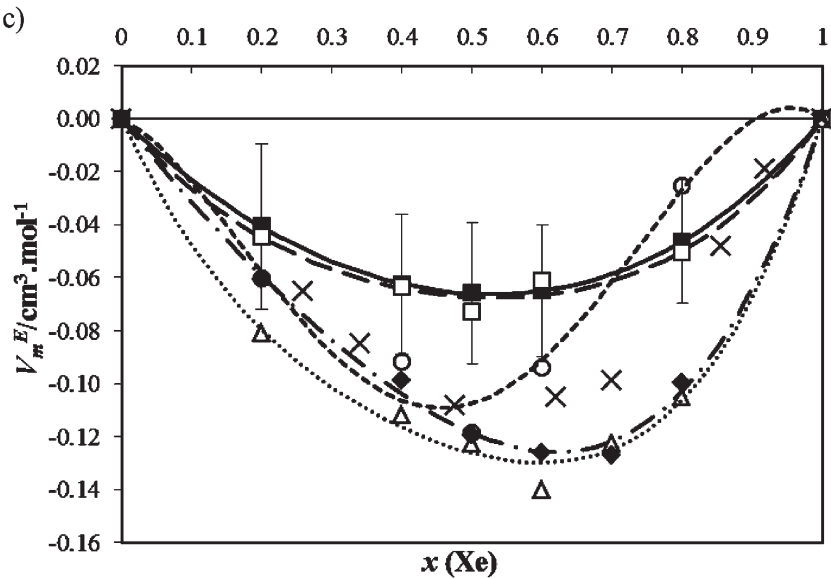

d)

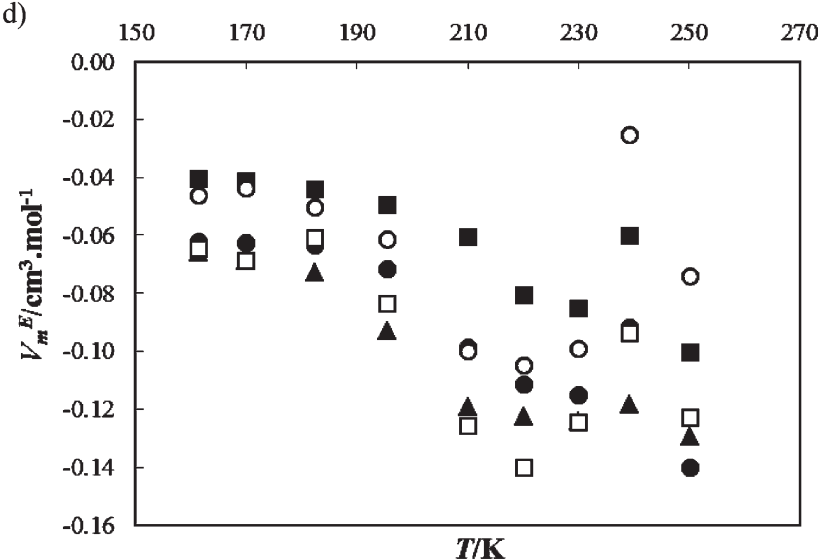

e)

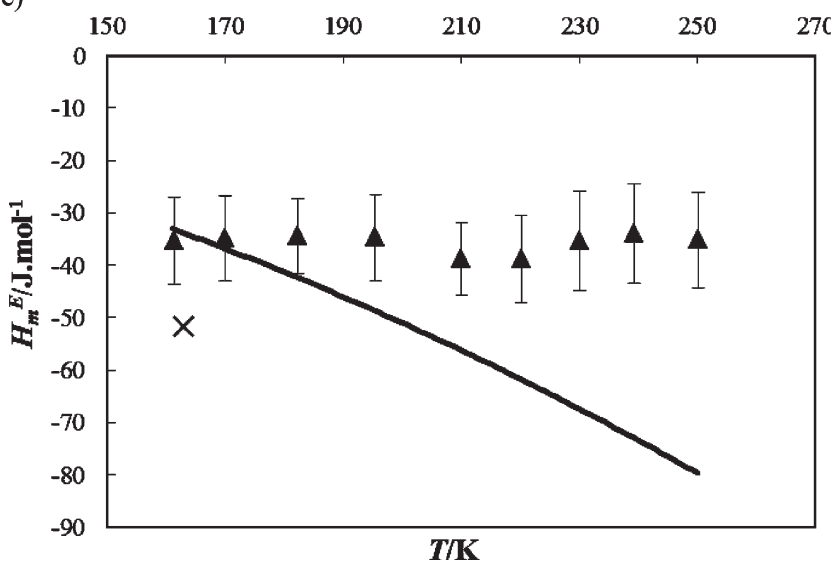

Figure 1. Excess functions for (xenon + ethane) system obtained by computer simulation. Excess molar enthalpies (a) as a function of xenon molar fraction for mixtures (points) and their Redlich-Kister correlations (lines) for several temperatures and (b) as a function of temperature at fixed compositions. Excess molar volumes (c) as a function of xenon molar fraction for mixtures (points) and their Redlich-Kister correlations (lines) for several temperatures and $(\mathrm{d}$ ) as a function of temperature at fixed compositions. (a, c) $161.40 \mathrm{~K},-$ and $\mathbf{\square}$; $182.34 \mathrm{~K},--$ and $\square$; $210.03 \mathrm{~K},-\cdots-$ and ४; $220.14 \mathrm{~K}, \cdots$ and $\Delta ; 239.3 \mathrm{~K},-$ - - and $\bigcirc$; $(\mathrm{b}, \mathrm{d}) x(\mathrm{Xe})=0.2, \mathbf{\square} ; x(\mathrm{Xe})=0.4, \mathbf{0} ; x(\mathrm{Xe})=0.5, \mathbf{\Delta} ; x(\mathrm{Xe})=0.6, \square$; $x(\mathrm{Xe})=0.8, \bigcirc($ lines, polynomial fittings to $\left.X_{\mathrm{m}}^{\mathrm{E}}\right)$. Crosses, experimental results at $163.0 \mathrm{~K}\left(H_{\mathrm{m}}^{\mathrm{E}}\right)$ or $161.4 \mathrm{~K}\left(V_{\mathrm{m}}^{\mathrm{E}}\right)$ from ref 23 . (e) Line, estimated curve of $H_{\mathrm{m}}^{\mathrm{E}}(x=0.5)$ from the experimental results of ref 32 .

compositions. Clearly, the point at which $X_{\mathrm{m}}^{\mathrm{E}}=0$ (enthalpy or volume for any composition) will be located beyond the lowest limit of our temperature range; that is, this implies an extrapolation to a temperature below the triple point of xenon. In the case of (xenon + ethane), the simulated excess enthalpies do not change with temperature, and for this reason, it was not possible to extrapolate the temperature at which $H_{\mathrm{m}}^{\mathrm{E}}$ becomes zero. For all the others, excess volume and enthalpy data as a function of temperature were fitted to polynomial functions, from which the temperature where $X_{\mathrm{m}}^{\mathrm{E}}=0$ was obtained by extrapolation. The 
a)

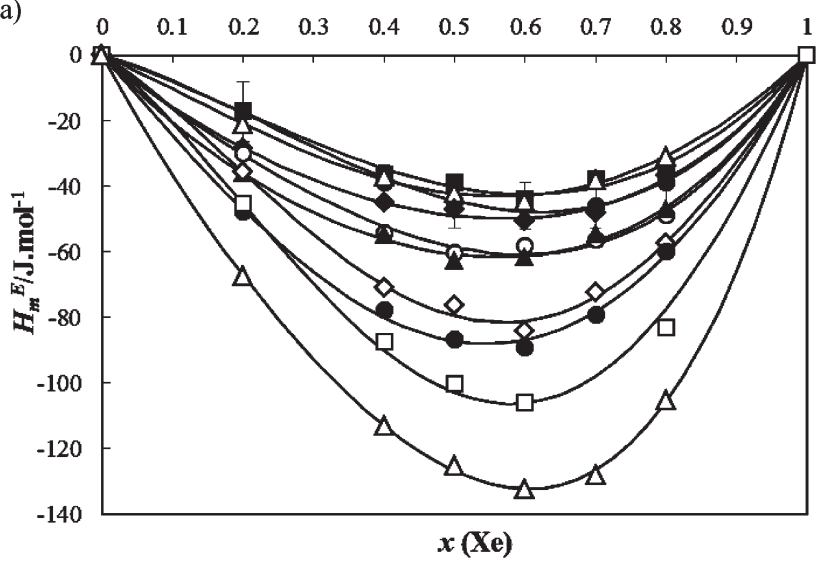

b) $\quad \begin{array}{rllllll}150 & 170 & 190 & 210 & 230 & 250 & 270\end{array}$

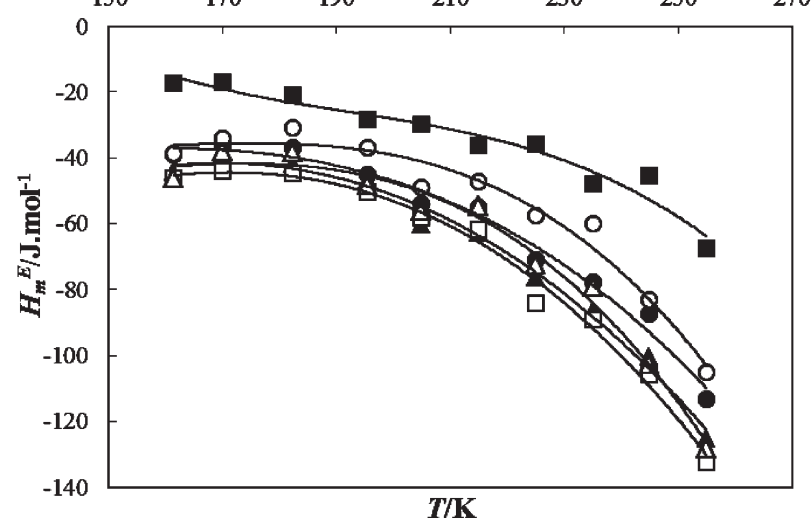

c)

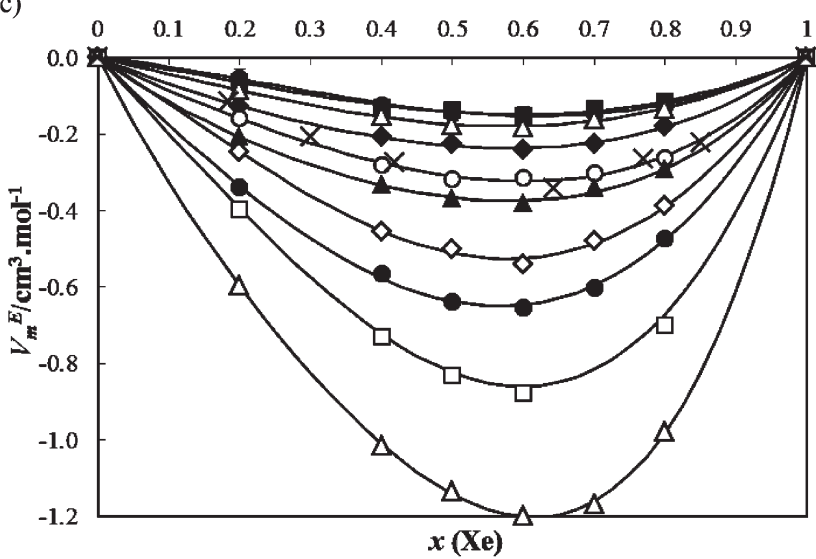

d)

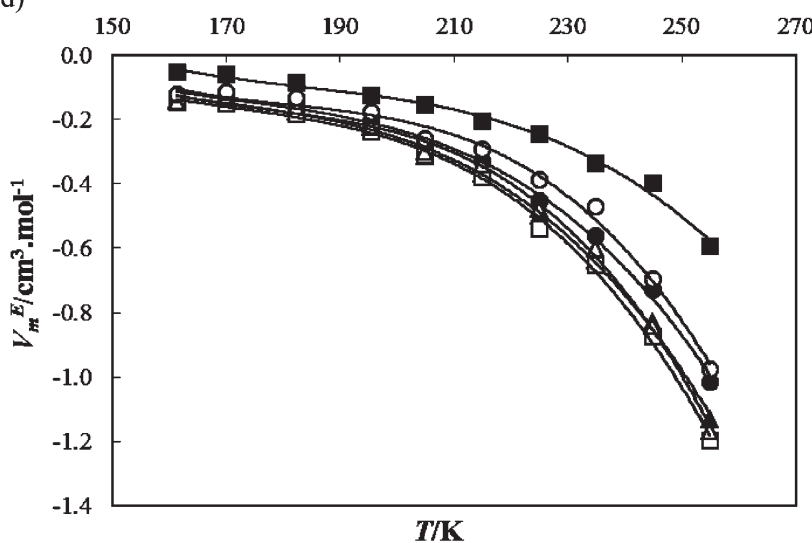

Figure 2. Excess functions for (xenon + propane) system obtained by computer simulation. Excess molar enthalpies (a) as a function of xenon molar fraction for mixtures (points) and their Redlich-Kister correlations (lines) for several temperatures and (b) as a function of temperature at fixed compositions. Excess molar volumes (c) as a function of xenon molar fraction for mixtures (points) and their Redlich-Kister correlations (lines) for several temperatures and (d) as a function of temperature at fixed compositions. (a, c) $161.40 \mathrm{~K}, \boldsymbol{\bullet} ; 170 \mathrm{~K}, \mathbf{\square} ; 182.34 \mathrm{~K}, \Delta$; $195.49 \mathrm{~K}, \boldsymbol{\nabla} ; 205 \mathrm{~K}, \mathrm{O} ; 215 \mathrm{~K}$, $\Delta ; 225 \mathrm{~K}, \diamond ; 235 \mathrm{~K}, \bullet ; 245 \mathrm{~K}, \square ; 255 \mathrm{~K}, \Delta .(\mathrm{b}, \mathrm{d}) x(\mathrm{Xe})=0.2$, $\mathbf{\bullet} ; x(\mathrm{Xe})=0.4, \boldsymbol{\bullet} ; x(\mathrm{Xe})=0.5, \boldsymbol{\Delta} ; x(\mathrm{Xe})=0.6, \square ; x(\mathrm{Xe})=0.7, \Delta ; x(\mathrm{Xe})=0.8, \bigcirc($ lines, polynomial fittings to $\left.X_{\mathrm{m}}^{\mathrm{E}}\right)$. Crosses, experimental results at $161.4 \mathrm{~K}$.

results are collected in Table 8 and, for (xenon + propane) and (xenon $+n$-butane), also shown in Figures 2 and 3 as solid lines. The reduced temperatures at which the properties vanish were also calculated using the average critical temperature $\left(T_{\mathrm{c} 12}\right)$ of each mixture, defined as ${ }^{36}$

$$
T_{\mathrm{c} 12}=\left(1-k_{12}\right)\left(T_{\mathrm{c} 1}-T_{\mathrm{c} 2}\right)^{1 / 2}
$$

where $T_{\mathrm{c} 1}$ and $T_{\mathrm{c} 2}$ are the critical temperatures of the pure components, and $k_{12}$ is a binary interaction parameter calculated by the expression from Tarakad and Danner, ${ }^{37}$

$$
1-k_{12}=\frac{8\left(V_{\mathrm{m}, \mathrm{cl}} V_{\mathrm{m}, \mathrm{c} 2}\right)^{1 / 2}}{\left(V_{\mathrm{m}, \mathrm{cl}}^{1 / 3}+V_{\mathrm{m}, \mathrm{c} 2}^{1 / 3}\right)^{3}}
$$

where $V_{\mathrm{m}, \mathrm{c} 1}$ and $V_{\mathrm{m}, \mathrm{c} 2}$ are the critical molar volumes of the pure components. Critical temperatures and molar volumes were taken from refs 38 (xenon) and 39 (ethane, propane, $n$-butane).

Despite the dispersion of the data, the temperature (and reduced temperature) at which $X_{\mathrm{m}}^{\mathrm{E}}=0$ is found to be remarkably constant over composition within each system. Furthermore, the calculated reduced temperature is almost constant from system to system, around $0.39-0.42$. This range is, however, quite different from that obtained for mixtures of $n$-alkanes $(0.57-0.59)$. This can be seen in Figure 4a,b where excess molar enthalpies and excess molar volumes at equimolar composition are plotted against reduced temperature for the three systems studied. For both properties, the three curves diverge at high reduced temperatures but seem to meet at a single convergence point at low temperatures. This seems to prove that mixtures of (xenon $+n$-alkanes) have a singularity point in their mixing properties when considered as a function of reduced temperature, similar to that exhibited by mixtures of $n$-alkanes. Again, if the extrapolations were done considering only the high temperature regime of the $H_{\mathrm{m}}^{\mathrm{E}}$ curves for both (xenon + propane) and (xenon $+n$-butane), the reduced temperatures at which $X_{\mathrm{m}}^{\mathrm{E}}=0$ would be closer to those of the $n$ alkane mixtures.

In Figure 5, we compare the $H_{\mathrm{m}}^{\mathrm{E}}$ and $V_{\mathrm{m}}^{\mathrm{E}}$ curves vs composition among the three systems studied at two different temperatures. At the same temperature, both excess functions decrease (to more negative values) as the alkane chain length increases. This is confirmed by experiment for $V_{\mathrm{m}}^{\mathrm{E}}$, and in the case of $H_{\mathrm{m}}^{\mathrm{E}}$, it is the trend experimentally found for mixtures of $n$-alkanes. Experimental values of $G_{\mathrm{m}}^{\mathrm{E}}$ for mixtures of (xenon $+n$-alkane) and ( $n$-alkane $+n$-alkane) also show this same behavior.

As previously explained, we have recently measured interaction enthalpies of dilute solutions of xenon in liquid $n$-alkanes and concluded that the interaction between xenon and the $\mathrm{CH}_{3}$ 
a)

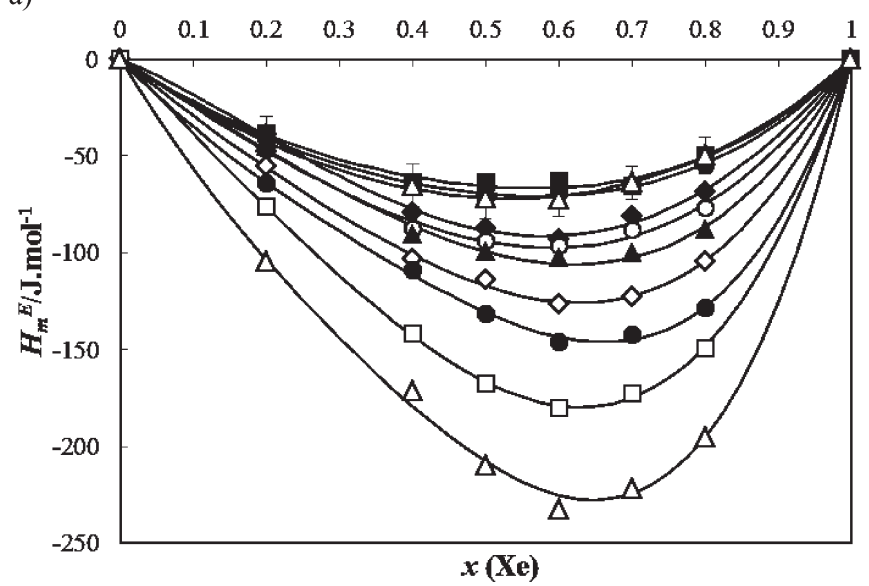

b) $\quad \begin{array}{lllllll}150 & 170 & 190 & 210 & 230 & 250 & 270\end{array}$

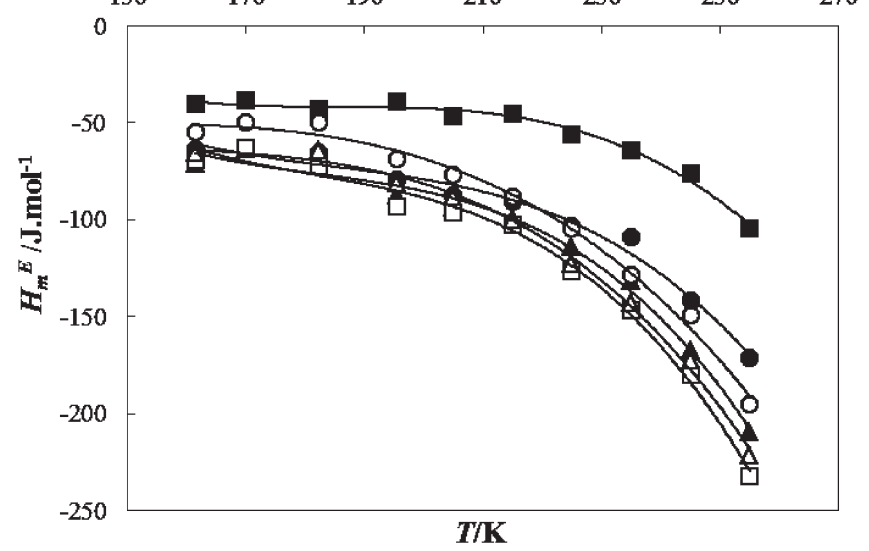

c)

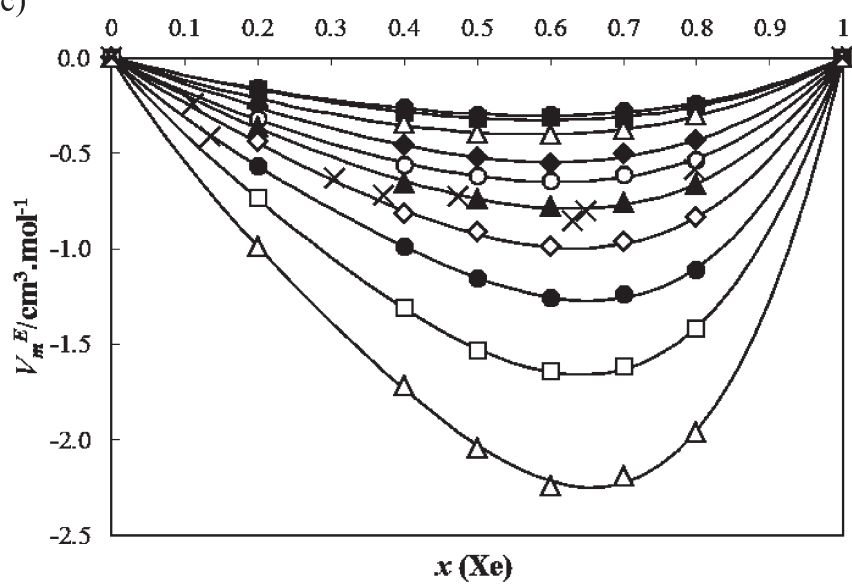

d)

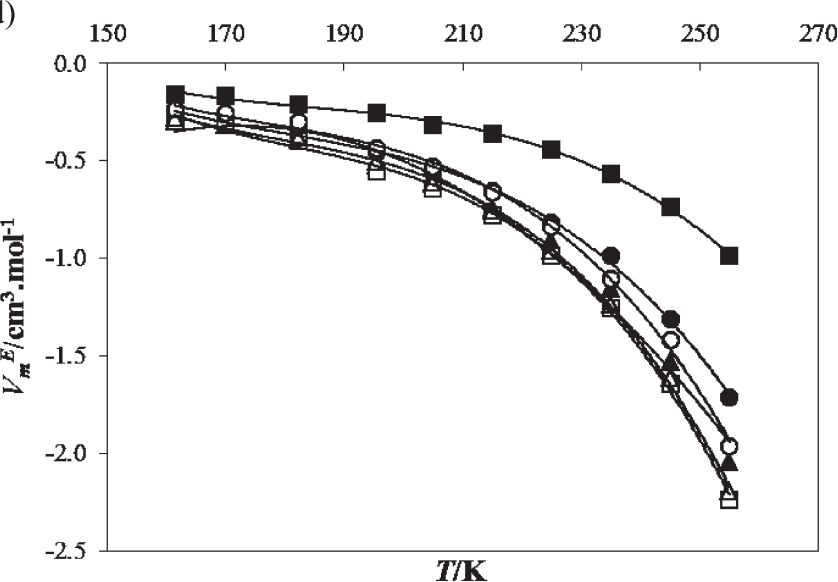

Figure 3. Excess functions for (xenon $+n$-butane) system obtained by computer simulation. Excess molar enthalpies (a) as a function of xenon molar fraction for mixtures (points) and their Redlich-Kister correlations (lines) for several temperatures and (b) as a function of temperature at fixed compositions. Excess molar volumes (c) as a function of xenon molar fraction for mixtures (points) and their Redlich-Kister correlations (lines) for several temperatures and $(\mathrm{d})$ as a function of temperature at fixed compositions. (a, b) $161.40 \mathrm{~K}, \boldsymbol{\bullet} ; 170 \mathrm{~K}, \mathbf{\square} ; 182.34 \mathrm{~K}, \Delta ; 195.49 \mathrm{~K}$, $; 205 \mathrm{~K}, \mathrm{O} ; 215 \mathrm{~K}$, $\Delta ; 225 \mathrm{~K}, \diamond ; 235 \mathrm{~K}, \boldsymbol{\bullet} ; 245 \mathrm{~K}, \square ; 255 \mathrm{~K}, \Delta .(\mathrm{b}, \mathrm{d}) x(\mathrm{Xe})=0.2, \mathbf{\square} ; x(\mathrm{Xe})=0.4, \boldsymbol{\Theta} ; x(\mathrm{Xe})=0.5, \mathbf{\Delta} ; x(\mathrm{Xe})=0.6, \square ; x(\mathrm{Xe})=0.7, \Delta ; x(\mathrm{Xe})=0.8, \bigcirc($ lines, polynomial fittings to $\left.X_{\mathrm{m}}^{\mathrm{E}}\right)$. Crosses, experimental results at $182.34 \mathrm{~K}$.

group is stronger than that with $\mathrm{CH}_{2} \cdot{ }^{27,28}$ This information is taken into account by the TraPPE-UA model used in this work; it uses a larger nonbonded energy parameter for $\mathrm{CH}_{3}$ than for $\mathrm{CH}_{2}$. This procedure, however, might seem to be in conflict with the simulation results because we have found that the excess enthalpy becomes more negative as the proportion of $\mathrm{CH}_{2}$ group within the alkane molecule increases. It is clear, however, that the absolute value of the excess enthalpy (as of all other excess functions) depends on the interactions not only between unlike molecules but also between like molecules and cannot be compared directly with interaction enthalpies of dilute solutions.

The appropriate way to rationalize the obtained results would be on a segment basis, rather a molar basis. One way to do this is through the application of the principle of congruence. The principle was initially proposed by Bronsted and Koefed ${ }^{40}$ and has been extensively used to interpret the thermodynamic behavior of mixtures of $n$-alkanes. According to the principle, the thermodynamic properties of the mixture are determined by the average chain length, which is given by

$$
n=\sum_{i} n_{i} x_{i}
$$

where $n_{i}$ is the number of carbon atoms in a molecule of the species $i$ and $x_{i}$ is the molar fraction of that species in the mixture. Mixtures with the same average chain length are said to be congruent. Hijmans ${ }^{41}$ devised a method for the application of principle of congruence to excess properties of binary mixtures, which involves the rescaling of the data to be comparable to a given reference system (usually the most asymmetric one) and expressing the properties as a function of the average chain length. The method has been applied to check the internal consistency of the different binary mixtures of $n$-alkanes. For a given binary system, any excess property as a function of $n$ would be obtained by

$$
\begin{aligned}
X_{\mathrm{m}}^{\mathrm{E}}\left(n, n_{1}, n_{2}\right)= & X_{\mathrm{m}}^{\mathrm{E}}\left(n, n_{A}, n_{B}\right)-\frac{n_{2}-n}{n_{2}-n_{1}} X_{\mathrm{m}}^{\mathrm{E}}\left(n_{1}, n_{A}, n_{B}\right) \\
& -\frac{n-n_{1}}{n_{2}-n_{1}} X_{\mathrm{m}}^{\mathrm{E}}\left(n_{2}, n_{A}, n_{B}\right)
\end{aligned}
$$

where $n_{1}$ and $n_{2}$ are the chain length of the pure components of the system; $n_{A}$ and $n_{B}$ are the chain lengths of the pure components of the reference system; and $X_{\mathrm{m}}^{\mathrm{E}}\left(n, n_{A}, n_{B}\right), X_{\mathrm{m}}^{\mathrm{E}}\left(n_{1}, n_{A}, n_{B}\right)$, and $X_{\mathrm{m}}^{\mathrm{E}}\left(n_{2}, n_{A}, n_{B}\right)$ are the values of excess property of the reference system for average chain lengths of $n, n_{1}$, and $n_{2}$, respectively. The 
Table 8. Values of Temperature and Reduced Temperature at Which the Excess Property (molar enthalpy and molar volume) Vanishes for (Xenon + Ethane), (Xenon + Propane) and (Xenon $+n$-Butane)

\begin{tabular}{|c|c|c|c|c|}
\hline$x_{\mathrm{Xe}}$ & $T\left(H_{\mathrm{m}}^{\mathrm{E}}=0\right) / \mathrm{K}$ & $T_{\mathrm{r}}\left(H_{\mathrm{m}}^{\mathrm{E}}=0\right)$ & $T\left(V_{\mathrm{m}}^{\mathrm{E}}=0\right) / \mathrm{K}$ & $T_{\mathrm{r}}\left(V_{\mathrm{m}}^{\mathrm{E}}=0\right)$ \\
\hline \multicolumn{5}{|c|}{ Xenon + Ethane } \\
\hline 0.2 & & & 102.4 & 0.345 \\
\hline 0.4 & & & 92.9 & 0.313 \\
\hline 0.5 & & & 79.7 & 0.268 \\
\hline 0.6 & & & 70.8 & 0.239 \\
\hline \multicolumn{5}{|l|}{0.8} \\
\hline \multicolumn{5}{|c|}{ Xenon + Propane } \\
\hline 0.2 & 134.8 & 0.417 & 147.9 & 0.457 \\
\hline 0.4 & 126.9 & 0.392 & 137.4 & 0.425 \\
\hline 0.5 & 127.8 & 0.395 & 133.0 & 0.411 \\
\hline 0.6 & 127.4 & 0.394 & 133.9 & 0.414 \\
\hline 0.7 & 129.9 & 0.401 & 140.3 & 0.434 \\
\hline 0.8 & 126.2 & 0.390 & 146.0 & 0.451 \\
\hline \multicolumn{5}{|c|}{ Xenon $+n$-Butane } \\
\hline 0.2 & 111.0 & 0.324 & 137.4 & 0.401 \\
\hline 0.4 & 114.7 & 0.335 & 139.5 & 0.407 \\
\hline 0.5 & 126.7 & 0.370 & 141.7 & 0.414 \\
\hline 0.6 & 134.7 & 0.393 & 143.8 & 0.420 \\
\hline 0.7 & 140.3 & 0.410 & 141.5 & 0.413 \\
\hline 0.8 & 140.8 & 0.411 & 142.1 & 0.415 \\
\hline
\end{tabular}

principle of congruence has been successfully applied to excess molar enthalpies, ${ }^{42}$ excess molar volumes, ${ }^{39}$ and excess Gibbs energies $^{43}$ for binary mixtures of $n$-alkanes having five carbon atoms or more. However, mixtures of lighter $n$-alkanes were found not to conform to the principle ${ }^{1}$ when systems involving methane were used as reference.

In this work, we have applied the principle of congruence to our excess molar enthalpy and excess molar volume data. In this approach, xenon was modeled as consisting of one segment. The results are shown in Figure 6 for excess molar enthalpies and in Figure 7 for excess molar volumes at four different temperatures. As can be seen from the figures, the overall picture is that the three systems approximately obey to the principle. In the case of excess molar enthalpies, both (xenon + ethane) and (xenon + propane) deviate from the curve of the reference system (xenon + $n$-butane) to more negative values of $H_{\mathrm{m}}^{\mathrm{E}}$ at the lowest temperatures, the deviations observed for (xenon + ethane) being more pronounced. We believe these deviations reflect the higher proportion of $\mathrm{CH}_{3}$ groups with which xenon interacts more strongly. As the temperature increases, the deviations decrease, probably as a consequence of thermal agitation, which tends to mask differences in the interactions. Regarding the excess volumes, it can be observed that (xenon + ethane) conforms to the (xenon $+n$-butane) results, but (xenon + propane) deviates to less negative volumes. Volume effects result from factors such as free volume and packing, which are obviously more difficult to interpret.

Solution Structure. The structure of the liquid mixtures was investigated through the analysis of radial distribution functions $\left(g_{\alpha \beta}(r)\right)$ at all temperatures. These were calculated for all pairs of interactive groups $\left(\mathrm{Xe}, \mathrm{CH}_{2}\right.$, and $\left.\mathrm{CH}_{3}\right)$ for the three systems at equimolar composition and also for the pure components.
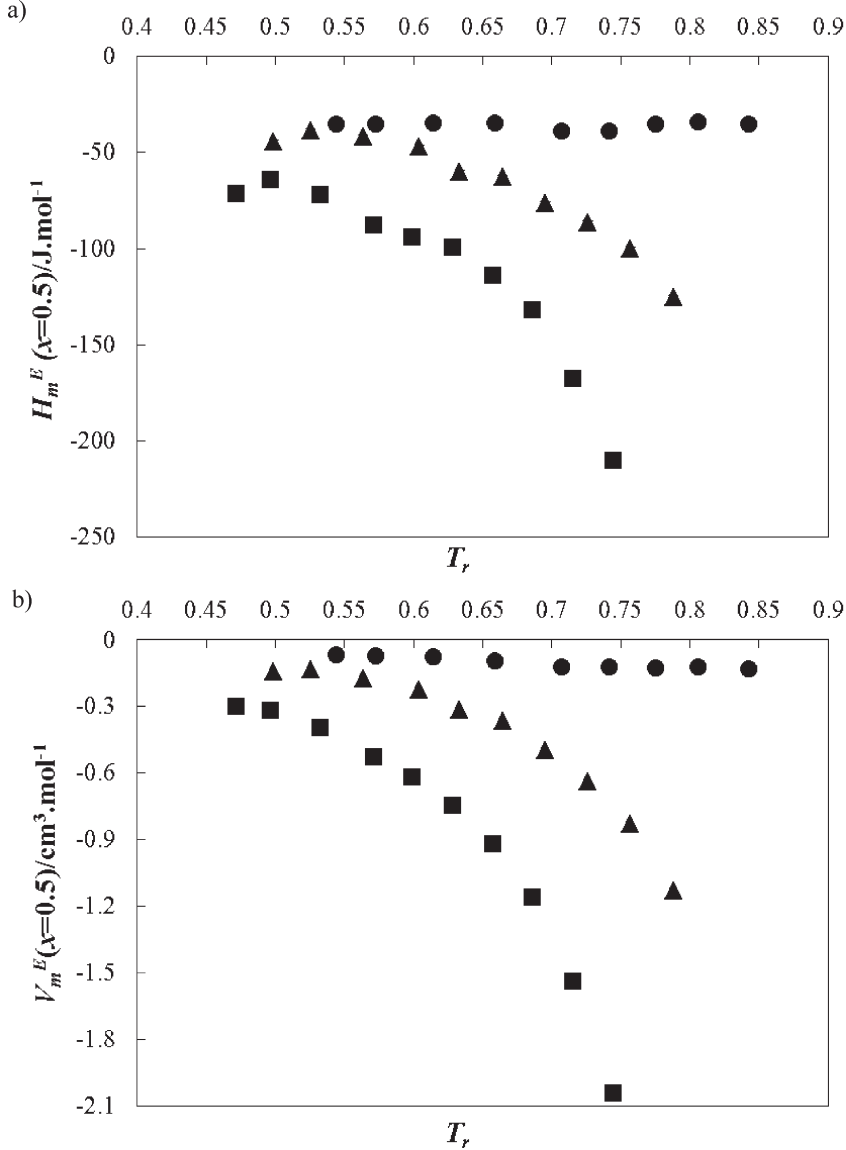

Figure 4. $H_{\mathrm{m}}^{\mathrm{E}}(x=0.5)(\mathrm{a})$ and $V_{\mathrm{m}}^{\mathrm{E}}(x=0.5)(\mathrm{b})$ for (xenon + ethane) $(\mathbf{O})$, (xenon + propane) $(\mathbf{\Lambda})$, and (xenon $+n$-butane) $(\boldsymbol{\square})$ as a function of reduced temperature.

Additional determinations for xenon molar fractions of 0.2 and 0.8 were done at four temperatures.

Center-to-center radial distribution functions for each pair of interactive groups are shown in Figure 8 at four different temperatures for (xenon + ethane) equimolar mixtures. The $g_{\alpha \beta}(r)$ of the $\mathrm{CH}_{3}-\mathrm{CH}_{3}$ pair for pure ethane and $\mathrm{Xe}-\mathrm{Xe}$ for pure xenon are omitted, since they are practically indistinguishable from those for equimolar mixture.

The radial distribution functions of methyl groups around xenon reveal a typical short-range order curve with a relatively narrow first peak, corresponding to a first coordination shell, with a small shoulder corresponding to the second methyl. The shoulder becomes more and more pronounced as the temperature increases. As expected, the peaks broaden at higher temperatures, and the intensity of the first peak decreases, but its position $\left(r_{\max }\right)$ remains unchanged.

The presence of a second interaction group is much more apparent in $\mathrm{CH}_{3}-\mathrm{CH}_{3}$ radial distribution functions for both equimolar mixtures and pure ethane. The shoulder in the first peak is clearly visible, becoming more important as the temperature increases and being, at the highest temperatures, as intense as the main peak. The position of the first peak slightly increases with temperature, whereas the intensity $\left(g_{\max }\right)$ decreases.

These findings are compatible with a predominance of perpendicular orientations between ethane molecules ( $\mathrm{T}$ arrangement) at low temperatures (which corresponds to the same mean distance between the reference group and each group of the other 


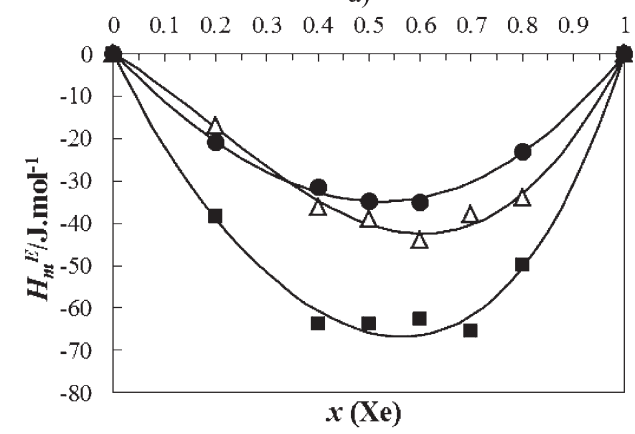

c)

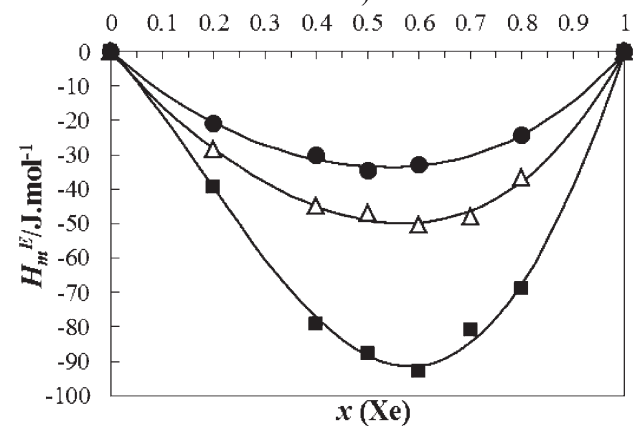

b)

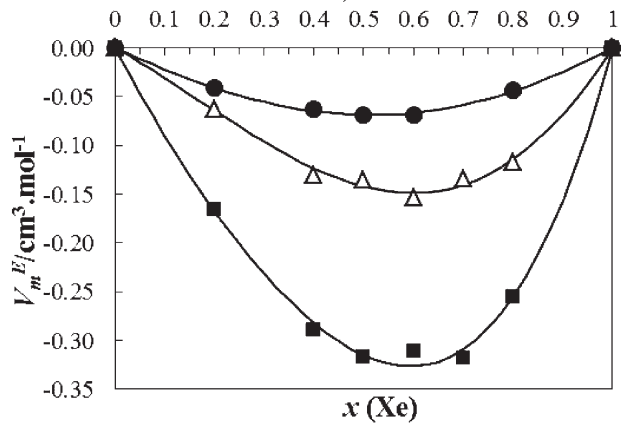

d)

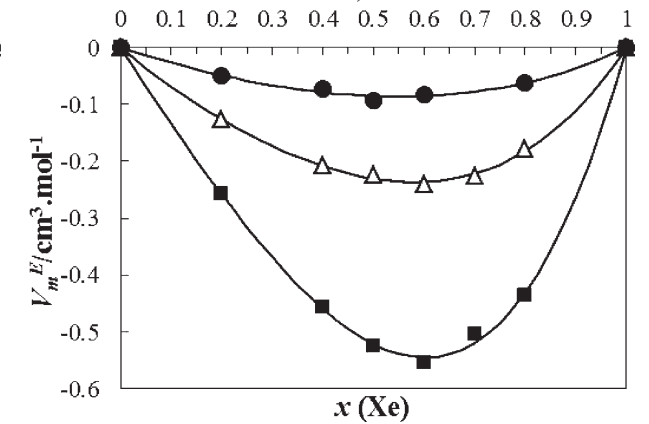

Figure 5. Excess properties as a function of xenon molar fraction for (xenon + ethane) $(\mathbf{O})$, (xenon + propane) $(\Delta)$, and (xenon $+n$-butane) $(\boldsymbol{\square})$ : (a) $H_{\mathrm{m}}^{\mathrm{E}}$ at $170 \mathrm{~K},(\mathrm{~b}) V_{\mathrm{m}}^{\mathrm{E}}$ at $170 \mathrm{~K}$, (c) $H_{\mathrm{m}}^{\mathrm{E}}$ at $195.49 \mathrm{~K}$, and (d) $V_{\mathrm{m}}^{\mathrm{E}}$ at $195.49 \mathrm{~K}$.
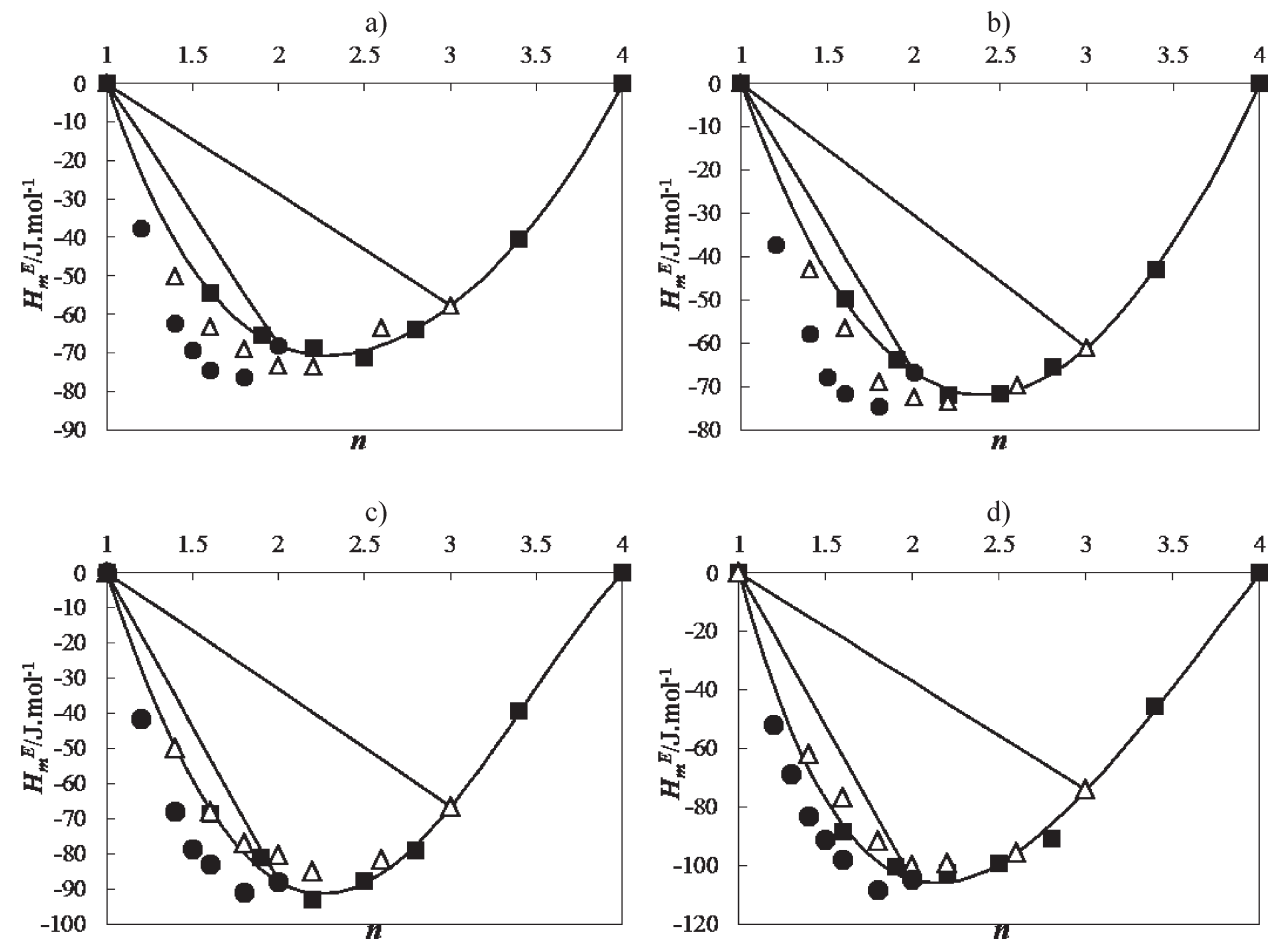

Figure 6. Excess molar enthalpies as a function of average chain length for (xenon + ethane) $(\mathbf{O})$, (xenon + propane) $(\Delta)$, and (xenon $+n$-butane) at four different temperatures: (a) 161.4, (b) 182.34, (c) 195.49, and (d) $235 \mathrm{~K}$ (xenon + ethane at $239.3 \mathrm{~K}$ ).

molecule). At higher temperatures, due to thermal agitation, more orientations would be accessible, in particular, in-line arrangements that contribute to the relative increase of the shoulder intensity. Similar considerations can be drawn for the $\mathrm{Xe}-\mathrm{CH}_{3}$ interaction, the effect being less pronounced because of the higher values of $\varepsilon$ and $\sigma$ for xenon.

$\mathrm{Xe}-\mathrm{Xe}$ seems to be the most "structured" interaction, with the most intense peaks, showing little influence of composition. 

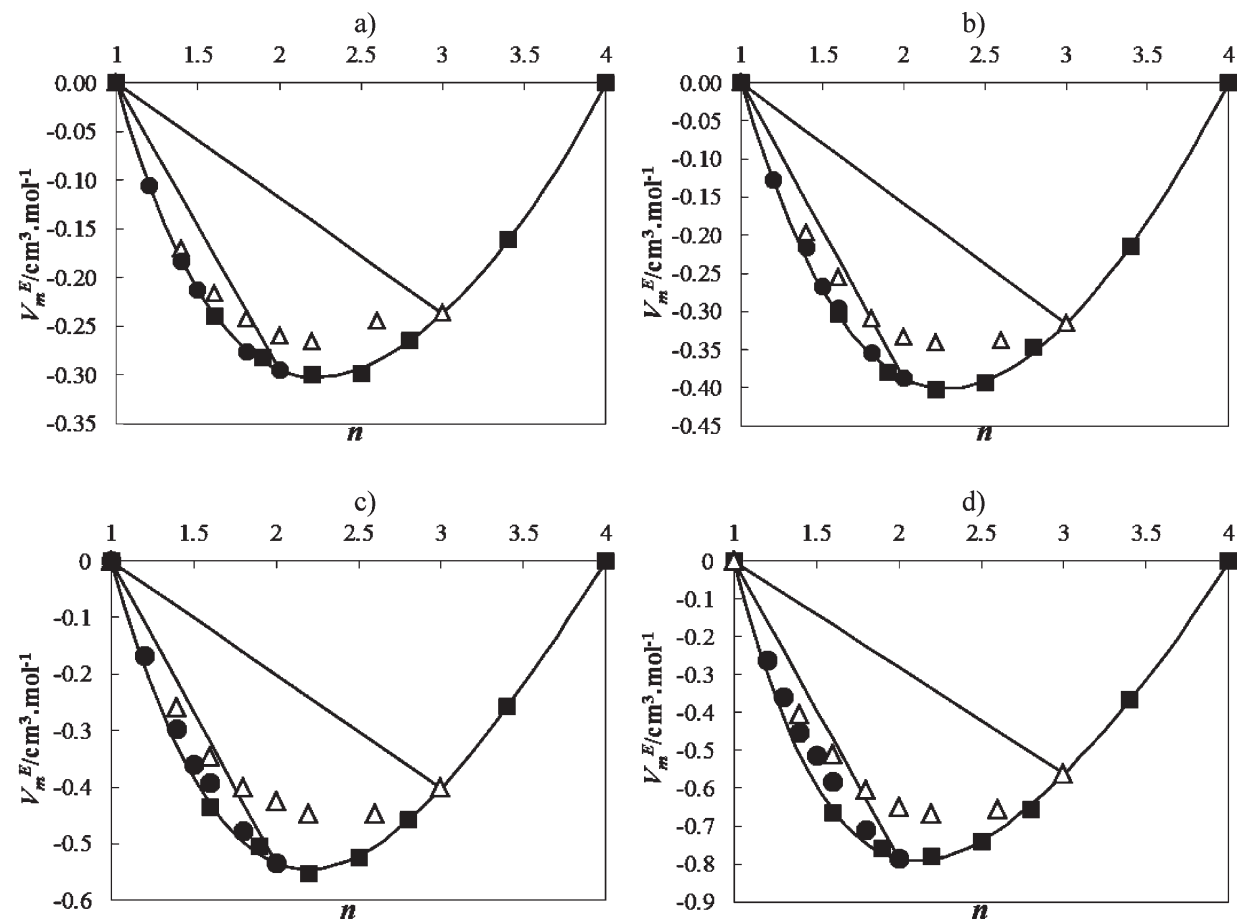

Figure 7. Excess molar volumes as a function of average chain length for (xenon + ethane) $(\bullet)$, (xenon + propane $)(\Delta)$, and (xenon $+n$-butane) at four different temperatures: (a) 161.4, (b) 182.34, (c) 195.49, and (d) $235 \mathrm{~K}$ (xenon + ethane at $239.3 \mathrm{~K}$ ).

a)

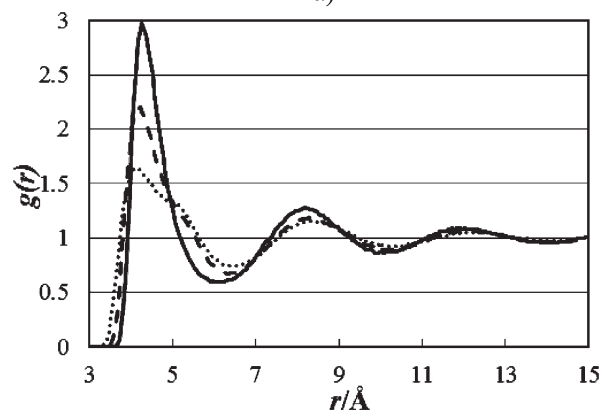

c)

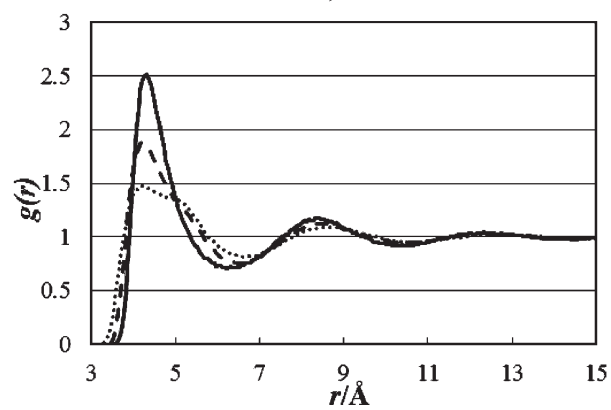

b)

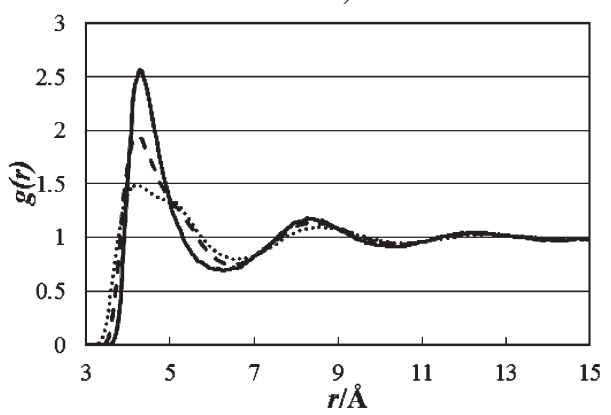

d)

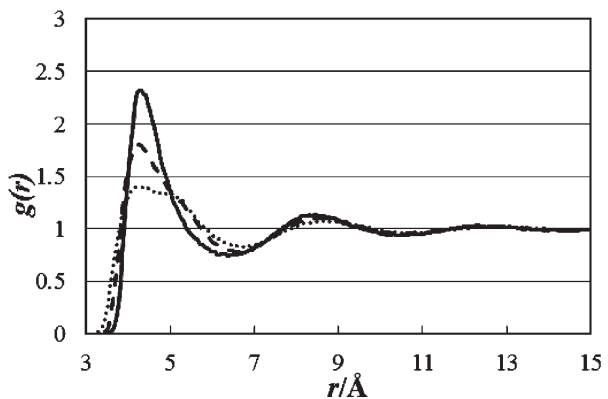

Figure 8. Radial distribution functions for (xenon + ethane) system at (a) 170, (b) 195.49, (c) 220.14, and (d) 239.3 K: Xe/ Xe, solid line; Xe/ $\mathrm{CH}_{3}$, dashed line; $\mathrm{CH}_{3} / \mathrm{CH}_{3}$, dotted line.

Its intensity decreases with the increasing temperature, but not its position. Finally, $r_{\max }$ for all the interaction pairs in (xenon + ethane) seems to be almost independent of composition, but decreases in the order $\mathrm{Xe}-\mathrm{Xe}>\mathrm{Xe}-\mathrm{CH}_{3}>\mathrm{CH}_{3}-\mathrm{CH}_{3}$; however, if the sizes of the groups are taken into account, this order is reversed. At higher temperatures, the differences in $r_{\max }$ disappear.

The radial distribution functions of all the possible pairs involving alkylic centers for equimolar mixtures of xenon with propane are shown in Figure 9 at four different temperatures. In 
a)

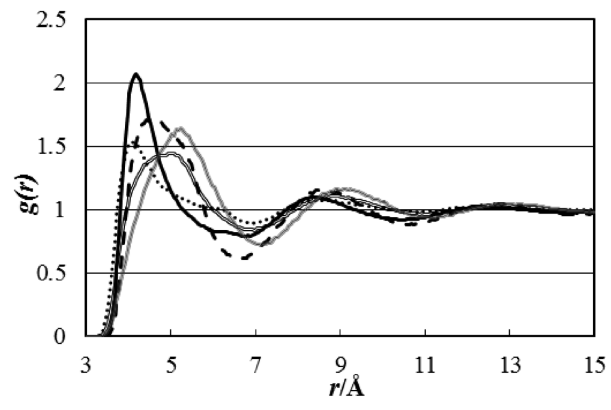

c)

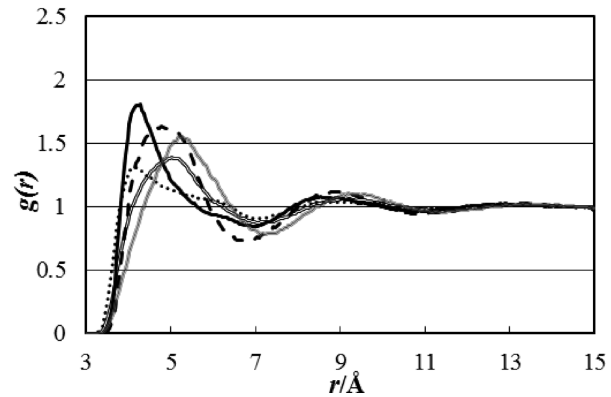

b)

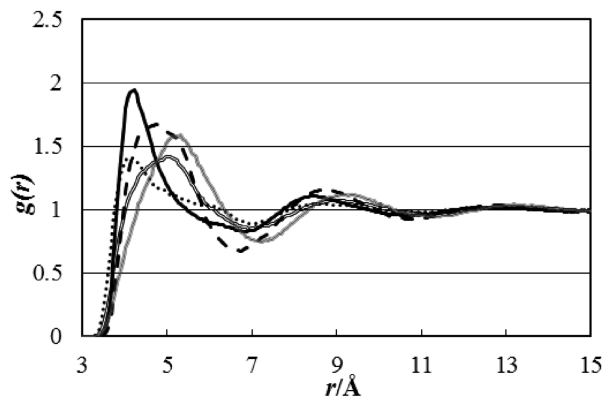

d)

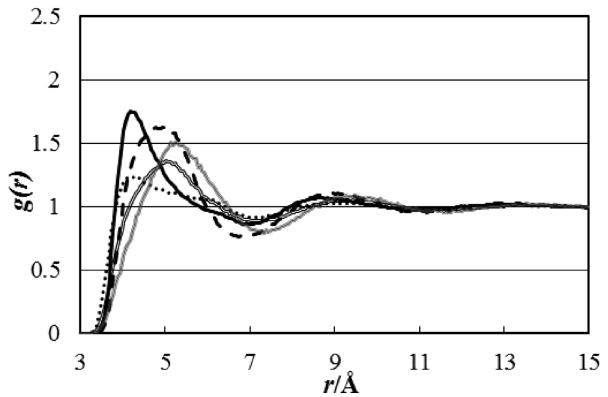

Figure 9. Radial distribution functions for interaction pairs involving alkylic groups in equimolar mixtures of (xenon + propane) system at (a) 170, (b) 195.49 , (c) 225 , and (d) $245 \mathrm{~K}: \mathrm{Xe}-\mathrm{CH}_{3}$, solid line; $\mathrm{Xe}--\mathrm{CH}_{2}$, dashed line; $\mathrm{CH}_{3}-\mathrm{CH}_{3}$, dotted line; $\mathrm{CH}_{3}-\mathrm{CH}_{2}$, double line; $\mathrm{CH}_{2}-\mathrm{CH}_{2}$, gray line.

a)

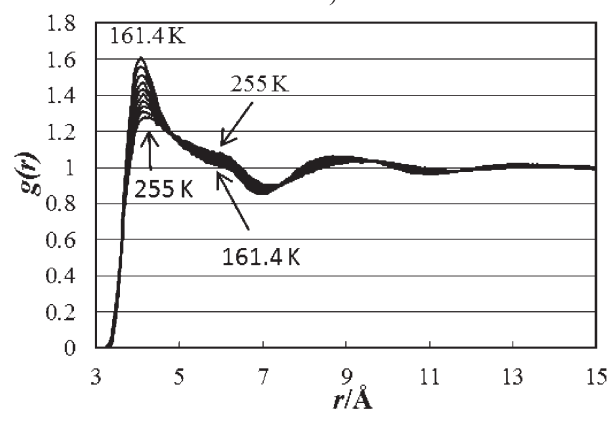

b)

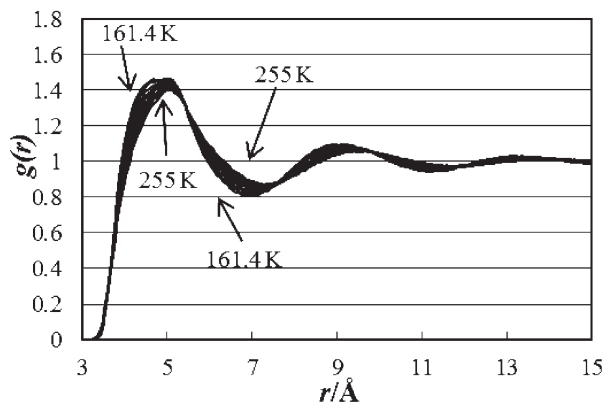

c)

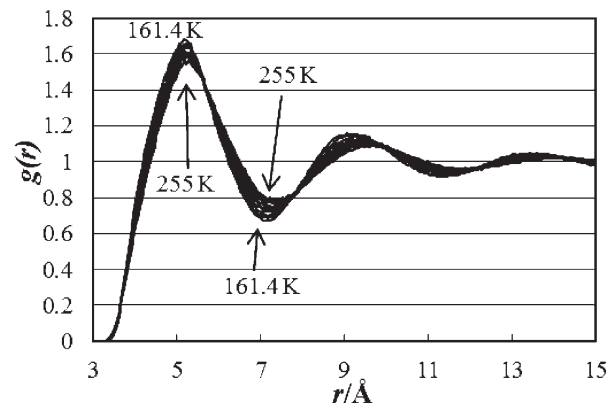

Figure 10. Radial distribution functions against temperature for pure propane: (a) $\mathrm{CH}_{3}-\mathrm{CH}_{3},(\mathrm{~b}) \mathrm{CH}_{3}-\mathrm{CH}_{2}$, and (c) $\mathrm{CH}_{2}-\mathrm{CH}_{2}$.

Figure 10, the curves for pure propane as a function of temperature are shown.

Again, the $\mathrm{Xe}-\mathrm{CH}_{3}$ peak is narrow, and the presence of the second methyl group is apparent only by a slight fluctuation of the baseline after the peak. As in (xenon + ethane), the $\mathrm{CH}_{3}-$ $\mathrm{CH}_{3}$ peak displays a shoulder, shifted to higher radial distances and becoming more pronounced with the increasing temperature. The first peaks for these two pairs are very different with respect to temperature dependence. For $\mathrm{CH}_{3}-\mathrm{CH}_{3}$, the intensity of the first peak decreases with increasing temperature, whereas the position slightly increases. In the case of $\mathrm{Xe}-\mathrm{CH}_{3}$, the peak maximum decreases with the 
a)

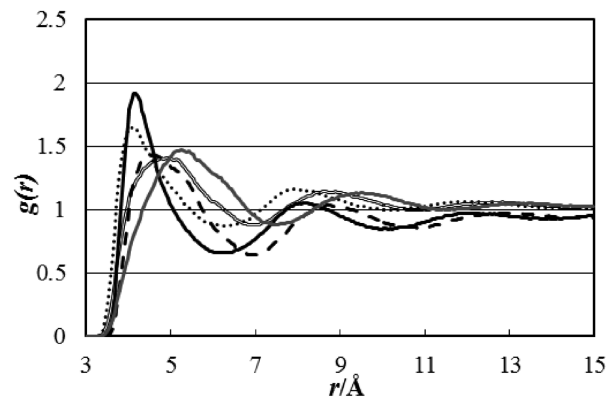

c)

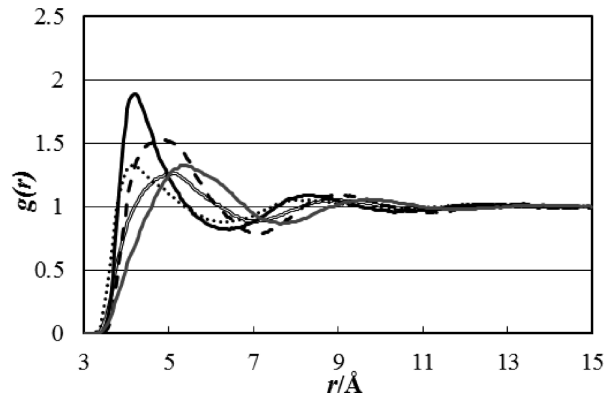

b)

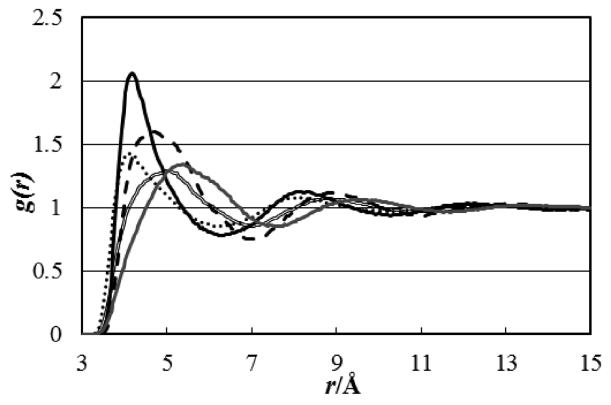

d)

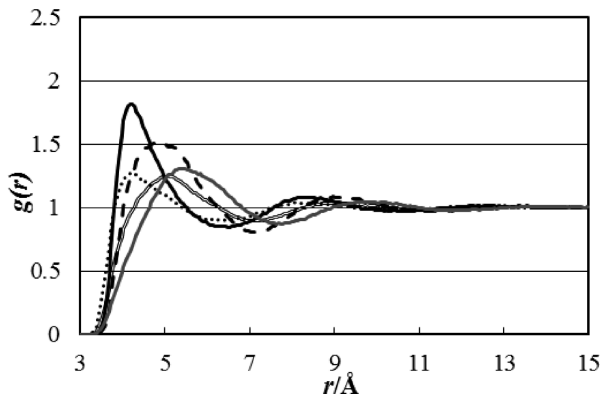

Figure 11. Radial distribution functions for interaction pairs involving alkylic groups in equimolar mixtures of (xenon $+n$-butane) system at (a) 170 , (b) 195.49 , (c) 225 , and (d) $245 \mathrm{~K}: \mathrm{Xe}-\mathrm{CH}_{3}$, solid line; $\mathrm{Xe}-\mathrm{CH}_{2}$, dashed line; $\mathrm{CH}_{3}-\mathrm{CH}_{3}$, dotted line; $\mathrm{CH}_{3}-\mathrm{CH}_{2}$, double line; $\mathrm{CH}_{2}-\mathrm{CH}_{2}$, gray line.

a)

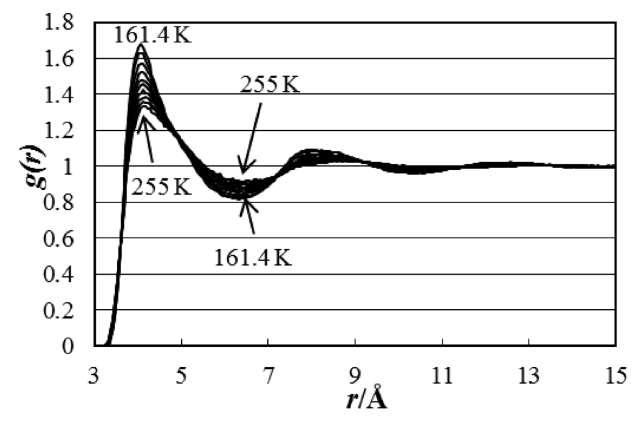

b)

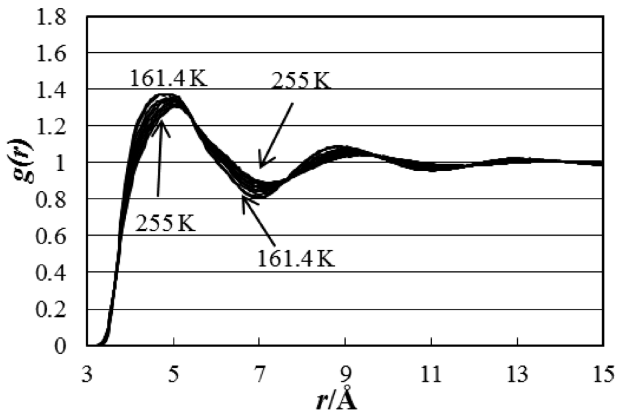

c)

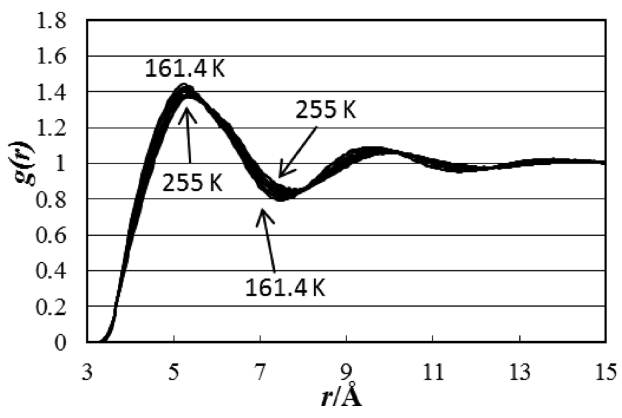

Figure 12. Radial distribution functions against temperature for pure $n$-butane: (a) $\mathrm{CH}_{3}-\mathrm{CH}_{3}$, (b) $\mathrm{CH}_{3}-\mathrm{CH}_{2}$, and (c) $\mathrm{CH}_{2}-\mathrm{CH}_{2}$.

increasing temperature, and $r_{\max }$ is practically independent of temperature.

The $g_{\alpha \beta}(r)$ for the $\mathrm{Xe}-\mathrm{CH}_{2}$ interaction is quite different from the previous pair. The first peak is smaller and broader than that of $\mathrm{Xe}-\mathrm{CH}_{3} ; g_{\max }$ decreases and $r_{\max }$ increases with the increasing temperature. In addition, $r_{\max }$ for the first peak of $\mathrm{Xe}-\mathrm{CH}_{2}$ is systematically higher than that of $\mathrm{Xe}-\mathrm{CH}_{3}$. Again, if the sizes of the groups are taken into account (using the segment diameter parameter of the TraPPE-UA model, $\sigma$ ) it is possible to calculate the "surface-to-surface" distance between groups, which is a better measure of their proximity because it does not depend on the group size. It is found that the "surface-to-surface" $\mathrm{Xe}-\mathrm{CH}_{2}$ distance is also larger than $\mathrm{Xe}-\mathrm{CH}_{3}$ and that difference increases with temperature. This can be an indication that 


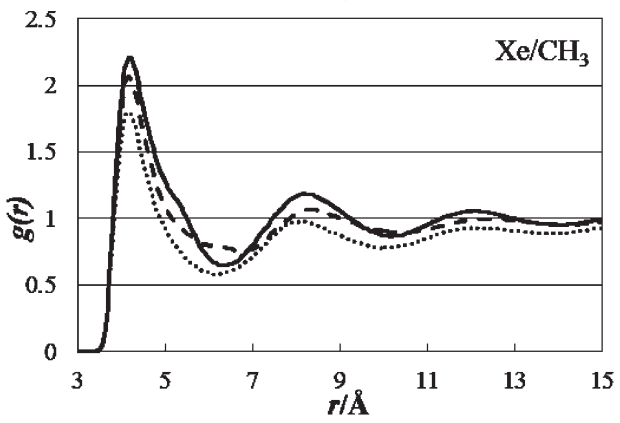

c)

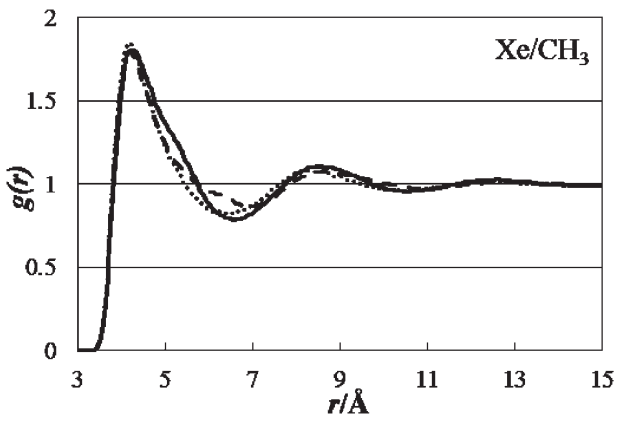

e)

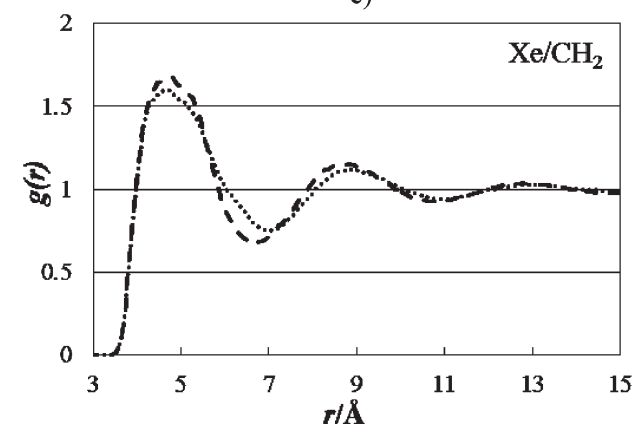

b)

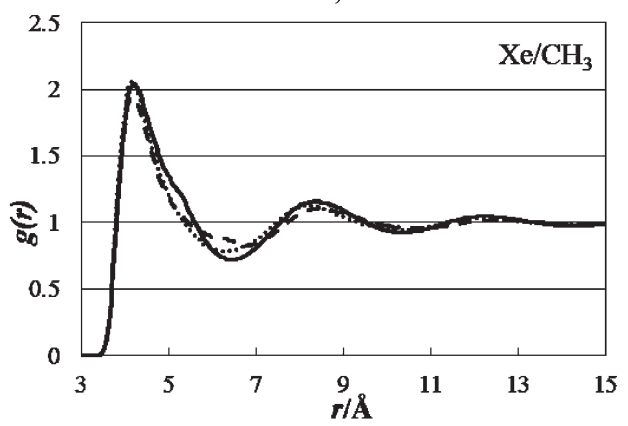

d)

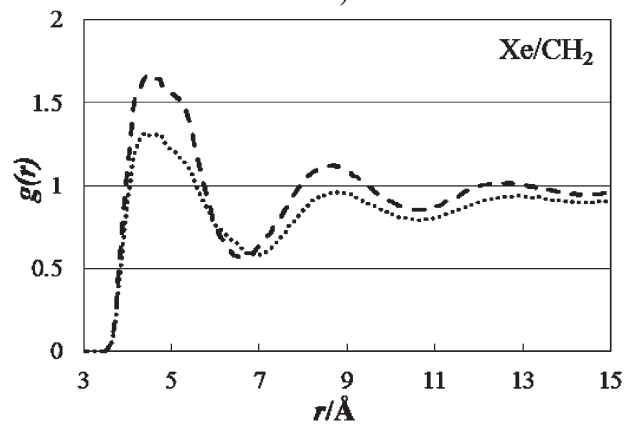

f)

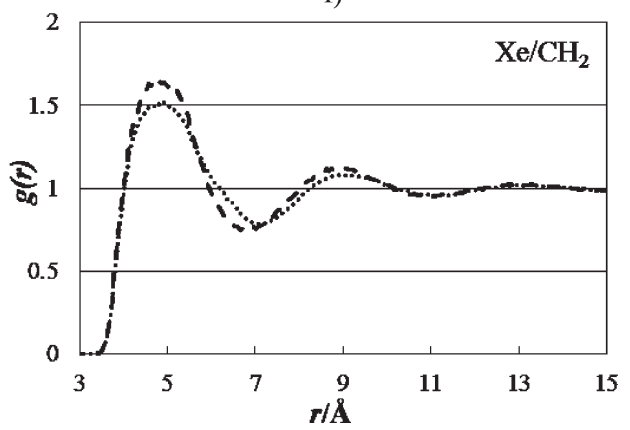

Figure 13. Radial distribution functions for (a) $\mathrm{Xe}-\mathrm{CH}_{3}$ at $161.4 \mathrm{~K}$, (b) $\mathrm{Xe}-\mathrm{CH}_{3}$ at $195.49 \mathrm{~K}$, (c) $\mathrm{Xe}-\mathrm{CH}_{3}$ at $235 \mathrm{~K}$ (xenon + ethane at $239.3 \mathrm{~K}$ ), (d) $\mathrm{Xe}-\mathrm{CH}_{2}$ at $161.4 \mathrm{~K}$, (e) $\mathrm{Xe}-\mathrm{CH}_{2}$ at $195.49 \mathrm{~K}$, and (f) $\mathrm{Xe}-\mathrm{CH}_{2}$ at $235 \mathrm{~K}$ of xenon + ethane (solid line), xenon + propane (dashed line), and xenon $+n$-butane (dotted line).

xenon interacts with propane preferably through the two methyl groups. For the remaining pairs, the order of $r_{\max }$ is $\mathrm{CH}_{3}-\mathrm{CH}_{3} \ll$ $\mathrm{CH}_{3}-\mathrm{CH}_{2}<\mathrm{CH}_{2}-\mathrm{CH}_{2}$, even considering the differences in $\sigma$. It is also interesting to note that the $\mathrm{CH}_{3}-\mathrm{CH}_{3}$ "surface-to-surface" distance is almost the same as that of $\mathrm{Xe}-\mathrm{CH}_{3}$.

For the (xenon $+n$-butane) system, the radial distribution functions of the interaction pairs involving alkylic groups for its equimolar mixtures are shown in Figure 11 at four temperatures, and those for pure $n$-butane as a function of temperature are shown in Figure 12. Both the $\mathrm{Xe}-\mathrm{CH}_{3}$ and $\mathrm{Xe}-\mathrm{CH}_{2}$ first peaks are relatively narrow, and their intensity does not change monotonically with temperature (increases and then decreases). The position of the former is not affected by temperature, but that of the latter slightly decreases. As in (xenon + propane), the $\mathrm{Xe}-\mathrm{CH}_{2}$ surface-to-surface distance is larger than that of $\mathrm{Xe}-\mathrm{CH}_{3}$. The $\mathrm{Xe}-\mathrm{CH}_{2}$ first peak is broader and may be the result of two fused peaks corresponding to the two $\mathrm{CH}_{2}$ groups in the molecule. Its intensity increases with temperature, becoming similar to that of $\mathrm{Xe}-\mathrm{CH}_{3}$. Both peaks decrease as the xenon mole fraction increases, but $r_{\max }$ is almost independent of composition.

In Figure 13, the radial distribution functions of $\mathrm{Xe}-\mathrm{CH}_{3}$ and $\mathrm{Xe}-\mathrm{CH}_{2}$ are compared for the three systems at three temperatures. For both pairs, the intensity and $r_{\max }$ of the first peak follows the order ethane $>$ propane $>n$-butane, although these differences disappear at higher temperature. The mean distance between xenon and each akylic groups seems to decrease with the increasing alkane chain length. This is probably due to the increase in alkane flexibility for longer alkanes, allowing a better accommodation around xenon. The $r_{\max }$ values for the other interaction pairs follow the same trend except for $\mathrm{CH}_{2}-\mathrm{CH}_{2}$, both in binary mixtures and in pure alkane.

The number of groups $(N)$ in the first coordination shell of a given reference center was estimated by integrating the radial distribution function as

$$
N=4 \pi \int_{r_{1}}^{r_{2}} g(r) r^{2} \rho
$$


a)

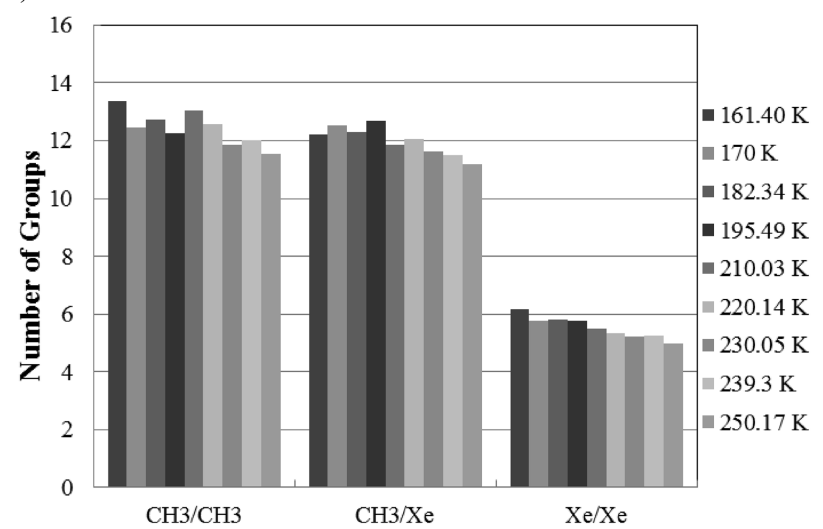

b)

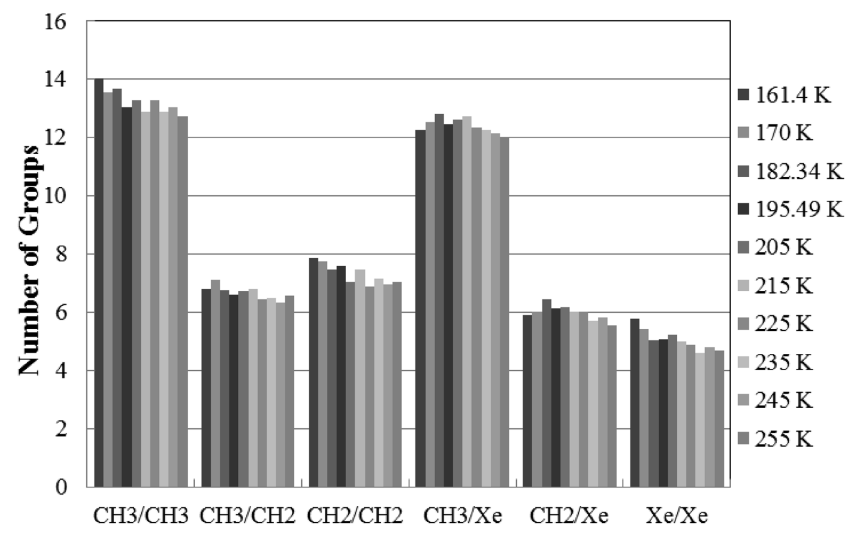

c)

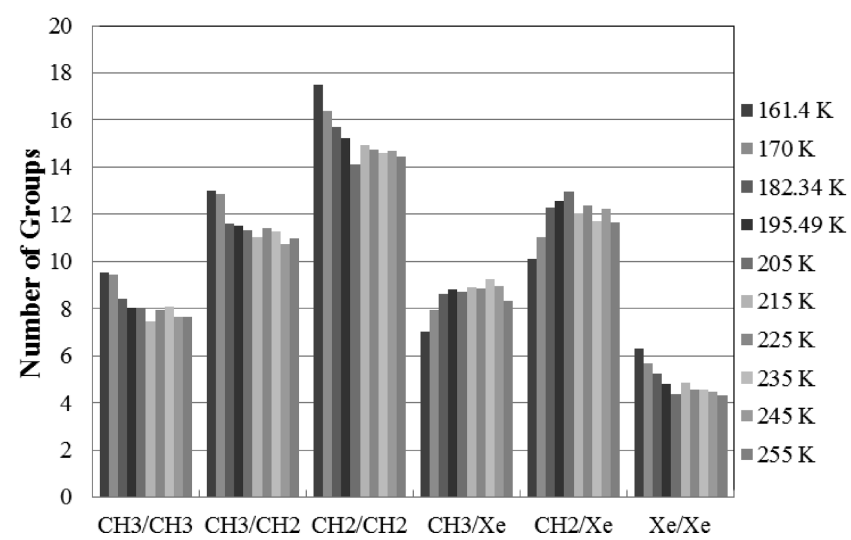

Figure 14. Estimated number of interaction groups around a reference center as a function of temperature for equimolar mixtures of (a) xenon + ethane (Xe- $\mathrm{CH}_{3}, \mathrm{CH}_{3}-\mathrm{CH}_{3}$, and $\mathrm{Xe}-\mathrm{Xe}$ pairs), (b) xenon + propane $\left(\mathrm{Xe}-\mathrm{CH}_{3}, \mathrm{Xe}-\mathrm{CH}_{2}, \mathrm{CH}_{3}-\mathrm{CH}_{3}, \mathrm{CH}_{3}-\mathrm{CH}_{2}, \mathrm{CH}_{2}-\mathrm{CH}_{2}, \mathrm{Xe}-\mathrm{Xe}\right.$ pairs), and (c) xenon $+n$-butane $\left(\mathrm{Xe}-\mathrm{CH}_{3}, \mathrm{Xe}-\mathrm{CH}_{2}, \mathrm{CH}_{3}-\mathrm{CH}_{3}\right.$, $\mathrm{CH}_{3}-\mathrm{CH}_{2}, \mathrm{CH}_{2}-\mathrm{CH}_{2}$, and $\mathrm{Xe}-\mathrm{Xe}$ pairs). For each block of columns, temperature is increasing from the left to the right.

where $r_{1}$ and $r_{2}$ are the inner and outer radii of the coordination shell and $\rho$ is the segment bulk density, which can be calculated from simulation results. In the calculations, $r_{1}$ is taken as the maximum $r$ for which $g_{\alpha \beta}(r)$ is zero before the first peak and $r_{2}$ is the $r$ corresponding to the first minimum of $g_{\alpha \beta}(r)$. The number of interaction groups around each reference group for the equimolar mixtures studied as a function of temperature is shown in Figure 14.
Table 9. Ratio between the Number of $\mathrm{CH}_{3}$ and $\mathrm{CH}_{2}$ Groups in the First Coordination Shell of Xenon for (Xenon + Propane $)$ and (Xenon $+n$-Butane $)$ at Four Different Temperatures and Extreme Compositions

\begin{tabular}{lccccc} 
& \multicolumn{4}{c}{$N_{\mathrm{CH}_{3}} / N_{\mathrm{CH}_{2}}$} \\
\cline { 2 - 3 }$T / \mathrm{K}$ & \multicolumn{2}{c}{ xenon + propane } & & xenon $+n$-butane \\
\cline { 2 - 3 } 170 & 2.054 & 2.000 & & 0.729 & 0.693 \\
195.49 & 2.175 & 2.114 & & 0.745 & 0.707 \\
225 & 2.105 & 2.084 & & 0.719 & 0.724 \\
245 & 2.009 & 2.007 & & 0.745 & 0.709 \\
\hline
\end{tabular}

In the case of (xenon + ethane) (Figure 14a), $N$ is similar for $\mathrm{Xe}-\mathrm{CH}_{3}$ and $\mathrm{CH}_{3}-\mathrm{CH}_{3}$ (average values of 12 and 12.4, respectively), but approximately one-half for $\mathrm{Xe}-\mathrm{Xe}$ (average value of 5.5) because the $\mathrm{Xe}-\mathrm{CH}_{3}$ first peak accounts for the two methyl groups per ethane molecule. This obviously reflects the fact that the molecular volume of xenon is approximately the same of that of the ethane molecules; thus, the double of the molecular volume of the methyl groups. $N$ also decreases as the temperature increases for the three interactive pairs. As stated above, in the case of the $\mathrm{Xe}-\mathrm{CH}_{3}$ pair, $r_{\max }$ is almost independent of temperature, despite the expansive effect caused by increasing the temperature. As can be seen here, expansion leads to a less populated first coordination shell, rather than an increasing of mean distance between centers.

In the case of (xenon + propane) (Figure 14b), it is found that the number of $\mathrm{CH}_{3}$ groups around a given xenon atom, relatively to the number of $\mathrm{CH}_{2}$ groups, is larger than what would be expected from their molecular proportion. This agrees with the differences, previously described, for the distances between the two types of pairs and can be seen as a further indication of a preferential interaction between xenon and $\mathrm{CH}_{3}$ relatively to $\mathrm{CH}_{2}$. A more efficient way of analyzing this effect is to calculate the ratio between the number of $\mathrm{CH}_{3}$ 's and the number of $\mathrm{CH}_{2}$ groups around xenon $\mathrm{N}_{\mathrm{CH}_{3}} / \mathrm{N}_{\mathrm{CH}_{2}}$, which in the case of random mixture of the components should be equal to 2 . These ratios were calculated as a function of mixture composition and are shown in Table 9 for extreme compositions. As can be seen, the ratio is always slightly larger than 2 and tends to be lower at higher xenon mole fractions. That fact seems to indicate that $\mathrm{CH}_{2}$ is more likely to populate the first coordination shell at low concentrations of alkane, where the alkylic groups are less available to xenon.

The integration of the radial distribution function first peak for (xenon $+n$-butane) was also calculated, and the results are included in Figure 14 (as a function of temperature), whereas the ratios $\mathrm{N}_{\mathrm{CH}_{3}} / \mathrm{N}_{\mathrm{CH}_{2}}$ can be found in Table 9 for the same compositions. In this case, $N_{\mathrm{CH}_{3}} / N_{\mathrm{CH}_{2}}$ for a random mixture should be 0.5 , since the second $\mathrm{CH}_{3}$ group of the $n$-butane molecule is, in principle, already outside the first coordination shell of xenon. As can be seen in Table 9, the ratios as a function of temperature and composition follow the same trend as for (xenon + propane): they are, in general, more favorable to $\mathrm{CH}_{2}$ at high xenon mole fraction than at low xenon concentration. Moreover, the effect seems to be more pronounced in (xenon + $n$-butane) than in (xenon + propane), which is consistent with the higher $\mathrm{CH}_{2}$ concentration in the former case. At low $n$-alkane 
concentrations, xenon seems to be less selective toward interacting with methyl and methylene groups.

Finally, it is interesting to note that the intensity of the $\mathrm{Xe}-\mathrm{Xe}$ peak in equimolar mixtures with different alkanes, decreases in the order $n$-butane $>$ propane $>$ ethane. This is surely due to a "dilution" effect: given the increasing molecular size and volume in the series ethane $<$ propane $<n$-butane, a $0.5 \mathrm{~mol}$ fraction corresponds to lower volume fractions of xenon. This is confirmed by integration of the first $g_{\alpha \beta}(r)$ peak, which showed a decreasing number of xenon atoms within its first coordination shell.

\section{CONCLUSIONS}

Excess molar enthalpies and excess molar volumes as a function of composition and temperature were obtained for binary mixtures of xenon with ethane, propane, and $n$-butane by computer simulation using the Monte Carlo method. The TraPPE-UA force field was used to model the light $n$-alkanes, and a simple LennardJones spherical potential was used to model xenon.

For all three systems, the simulation results predicted excess volumes in good agreement with the experimental data. The simulated excess enthalpies also agree with the experimental results for (xenon + ethane), confirming the weak dependence with temperature exhibited by this system. In the case of (xenon + propane $)$ and (xenon $+n$-butane), however, the simulation predicts negative excess enthalpies, and those estimated from experimental data are positive. Both excess volumes and enthalpies display a complex dependence with temperature: a slow varying region followed by a sharp decrease, which in some aspects resembles that found for mixtures of $n$-alkanes. At low temperature, the curves seem to converge to a common reduced temperature at which both properties vanish at all the compositions. This universal temperature is different from that previously found for $n$-alkane mixtures; however, if only the high temperature regime is used, the results may lead to a common reduced temperature at which $H_{\mathrm{m}}^{\mathrm{E}}=0$ in close agreement with that observed for mixtures of $n$-alkanes.

The structure of the liquid mixtures was investigated through the calculation of radial distribution functions for all interaction pairs over the same range of temperatures. We have found that the xenon-methyl distance is systematically shorter than that of xenon-methylene in all systems and at all compositions. An estimation of the number of groups in the first coordination shells was also obtained by numerical integration of the radial distribution functions' first peaks. It was found that the relative proportion of methyl and methylene groups around xenon, $\mathrm{N}_{\mathrm{CH}_{3}} / \mathrm{N}_{\mathrm{CH}_{2}}$, is systematically higher than that expected for a random mixture. This, combined with the observed distances between groups, seems to indicate that xenon's first coordination shell is preferentially more populated by $\mathrm{CH}_{3}$ than by $\mathrm{CH}_{2}$ groups.

\section{AUTHOR INFORMATION}

\section{Corresponding Author}

*E-mail: 1fgm@uevora.pt.

\section{ACKNOWLEDGMENT}

The authors acknowledge financial support from the Portuguese Fundação para a Ciência e a Tecnologia through Project POCTI/QUI/46299/2002 and technical facilities from Centro de Geofísica de Évora of University of Évora.

\section{REFERENCES}

(1) Shana'a, M. Y.; Canfield, F. B. Trans. Faraday Soc. 1968, 64, 2281-2286.

(2) Sanders, R. D.; Franks, N. P.; Maze, M. Br. J. Anasth. 2003, 91, 709-17.

(3) Harris, P. D.; Barnes, R. Anaesthesia 2008, 63, 284-293.

(4) Wlodarczyk, A.; McMillan, P. F.; Greenfield, S. A. Chem. Soc. Rev. 2006, 35, 890-898.

(5) Sanders, R. D.; Ma, D.; Maze, M. Br. Med. Bull. 2005, 71, 115.

(6) Tonner, P. H.; Bangert, K.; Scholz, J. Best Prac. Res. Clin. Anaesthesiol. 2001, 15 (3), 491-503.

(7) Preckel, B.; Schlack, W. Best Prac. Res. Clin. Anaesthesiol. 2005, 19 (3), 365-379.

(8) Hirota, K. Best Prac. Res. Clin. Anaesthesiol. 2006, 20 (1), 69-79.

(9) Lynch, C., III; Baum, J.; Tenbrinck, R. Anesthesiology 2000, $92(3), 865-868$.

(10) Trudell, J. R.; Koblin, D. D.; Eger, E. I., II. Anesth. Analg. 1998, 87, 411-418.

(11) Koblin, D. D.; Fang, Z.; Eger, E. I., II; Laster, M. J.; Gong, D.; Ionescu, P.; Halsey, M. J.; Trudell, J. R. Anesth. Analg. 1998, 87, 419-424.

(12) Franks, N. P.; Lieb, W. R. Nature 1987, 328, 113.

(13) Franks, N. P.; Lieb, W. R. Nature 1994, 367, 607-614.

(14) Franks, N. P.; Dickinson, R.; Sousa, S. L. M.; Hall, A. C.; Lieb, W. R. Nature 1998, 396, 324.

(15) Miller, K. W. Br. J. Anaesth. 2002, 89, 17-31.

(16) Rowlinson, J. S.; Swinton, F. L., Liquids and Liquid Mixtures, 3rd ed.; Butterworth Scientific: London, 1982.

(17) Marsh, K. N.; Ott, J. B.; Costigan, M. J. J. Chem. Thermodyn. 1980, 12, 343

(18) Marsh, K. N.; Ott, J. B.; Richards, A. E. J. Chem. Thermodyn. 1980, 12, 897 .

(19) Ott, J. B.; Marsh, K. N.; Stokes, R. H. J. Chem. Thermodyn. 1981, 13, 371 .

(20) Fernández-Garcia, J. G.; Boissonnas, Ch. Helv. Chim. Acta 1967, 106-107 (50), 4.

(21) Martins, L. F. G. Tese de Doutoramento; Instituto Superior Técnico: Lisboa, 1999.

(22) dos Ramos, M. C.; Blas, F. J. J. Phys. Chem. B 2005, 109, $12145-12153$.

(23) Filipe, E. J. M.; Gomes de Azevedo, E. J. S.; Martins, L. F. G.; Soares, V. A. M.; Calado, J. C. G.; McCabe, C.; Jackson, G. J. Phys. Chem. B 2000, 104, 1315-1321.

(24) Filipe, E. J. M.; Martins, L. F. G.; Calado, J. C. G.; McCabe, C.; Jackson, G. J. Phys. Chem. B 2000, 104, 1322-1325.

(25) Pata, C. I. S.; Filipe, E. J. M.; Martins, L. F. G. Unpublished results.

(26) Filipe, E. J. M.; Dias, L. M. B.; Calado, J. C. G.; McCabe, C.; Jackson, G. Phys. Chem. Chem. Phys. 2002, 4, 1618.

(27) Bonifácio, R. P. M. F.; Martins, L. F. G.; McCabe, C.; Filipe, E. J. M. J. Phys. Chem. B 2010, 114, 15897-15904.

(28) Bonifácio, R. P. M. F.; Filipe, E. F. M.; dos Ramos, M. C.; Blas, F. J.; Martins, L. F. G. Fluid Phase Equilib. 2011, 303, 193-200.

(29) Palace Carvalho, A. J.; Prates Ramalho, J. P.; Martins, L. F. G. J. Phys. Chem. B 2007, 102, 2569.

(30) Martin, M. G.; Siepmann, J. I. J. Phys. Chem. B 1998, 102, 2569.

(31) Bohn, M.; Lago, S.; Fischer, J.; Kohler, F. Fluid Phase Equilib. 1985, 23, 137.

(32) Nunes da Ponte, M.; Chokappa, D.; Calado, J. C. G.; Clancy, P.; Streett, W. B. J. Phys. Chem. 1985, 89, 2746.

(33) Gubbins, K. E.; Twu, C.-H. Chem. Eng. Sci. 1978, 10, 33.

(34) Vega, C.; MacDowell, L. G.; López-Rodriguez, A. J. Chem. Phys. 1999, 111, 3192-3202.

(35) Blas, F. J. J. Phys. Chem. B 2000, 104, 9239-9248.

(36) Poling, B.E.; Prausnitz, J. M.; O'Connell, J. P. The Properties of Gases and Liquids; McGraw-Hill: New York, 2000.

(37) Tarakad, R. R.; Danner, R. P. AIChE J. 1977, 23, 685.

(38) Habgood, H. W.; Schneider, W. G. Can. J. Chem. 1954, 32, 98. 
(39) Ambrose, D.; Walton, J. Pure Appl. Chem. 1989, 61 (8), 1395-1403.

(40) Bronsted, J. N.; Koefoed, J. Kgl. Danske Videnskab. Selskab., Mat.-fys. Medd. 1946, 22 (17), 1.

(41) Hijmans, J. Mol. Phys. 1958, 1, 307.

(42) Holleman, Th. Physica 1965, 31, 49.

(43) Weiguo, S.; Qin, A. X.; McElroy, P. J.; Williamson, A. G. J. Chem. Thermodyn. 1990, 22, 905. 